\title{
Assessment of a miniature four-roll mill and a cross-slot microchannel for high-strain-rate stagnation point flows
}

\author{
Farzan Akbaridoust*, Jimmy Philip, and Ivan Marusic \\ Department of Mechanical Engineering, University of Melbourne, Victoria 3010, Australia \\ *E-mail: farzan.akbaridoust@unimelb.edu.au
}

\begin{abstract}
Stagnation point flows have been widely used to study the deformation and break-up of objects in two-dimensional pure straining flows. Here, we report a systematic study of the characterisation of stagnation point flows in two devices, a miniature Taylor's four-roll mill and a cross-slot microchannel. The aim of the study is to find the best platform suitable for investigating the effect of strain rate on the mechanical properties of waterborne microorganisms. Using micro-PIV, the velocity field and the strain rates in both devices were measured at different flow rates and compared with an ideal hyperbolic stagnation point flow. The cross-slot microchannel was found to be a better experimental device than the miniature four-roll mill for the purpose of confining micron-sized objects in a controlled stagnation point flow. This is mainly due to the difficulty of maintaining a fixed location for the stagnation point within one micron in the miniature four-roll mill and achieving high strain rates beyond $10 \mathrm{~s}^{-1}$. However, with no moving parts, the cross-slot microchannel was found to maintain a steady flow with the stagnation point varying less than one micron at a cross-junction of $400 \times 400 \mu \mathrm{m}^{2}$, and was able to reach uniform strain rates up to $140 \mathrm{~s}^{-1}$.
\end{abstract}

Keywords: microfluidics, micro-PIV, stagnation point, extensional flow, high strain rate flows, cross-slot microchannel, four-roll mill

\section{Introduction}

Stretching objects in extensional fluid flows and studying their deformation and breakage have been widely carried out during the last century. Different techniques exist to enable one to produce extensional flows. These include changing the crosssectional area of the flow by contraction or expansion [1], thinning fluid filaments using jetting or dripping [2], and bifurcating the flow [3] using two opposed laminar streams (stagnation point flow). Stagnation point flow has the advantage of providing a spatially uniform strain rate. Moreover, it enables one to study the deformation of an object at a certain fixed location (i.e. at the stagnation point).

In the present study we are interested in providing a platform for trapping microorganisms at the stagnation point and subjecting them to high strain rates with water based solutions as a medium. This enables one to investigate the effect of the strain rate on cyanobacteria, which is the cause of significant water contamination issues worldwide. For this purpose we assess two devices, a miniature four-roll mill and a cross slot microchannel. In the following we provide a brief overview of these devices.

\section{$1.1 \quad$ Four-roll mill}

The most common apparatus to produce an extensional stagnation point flow is a four-roll mill. A four-roll mill is a device that contains four cylindrical rollers in a fluid container (schematically shown in figure 1). Each roller axis is set parallel to each other symmetrically in a square layout. The four- 
roll mill was invented by G. I. Taylor in 1934 to investigate the deformation of fluid droplets [3]. By systematic direction of the rotation of the rollers (as shown in the top image in figure 1), a pure extensional stagnation point flow can be produced in a four-roll mill and the velocity equations are given by:

$$
\begin{gathered}
u=\gamma x, \\
v=-\gamma y,
\end{gathered}
$$

where $\gamma$ is the strain rate of the flow. Pure extensional stagnation point flow has a zerovelocity/stagnation point $(x=y=0$ in figure 1$)$, and linear velocity increments with distance $x$ and $y$. The flow has hyperbolic streamlines $(x . y=$ const.) and spatially uniform stain rate $(\gamma=$ const.). An object that is placed in the stagnation point experiences an attractive force along the compressional axis, whilst it experiences a repulsive force along the extensional axis [4].

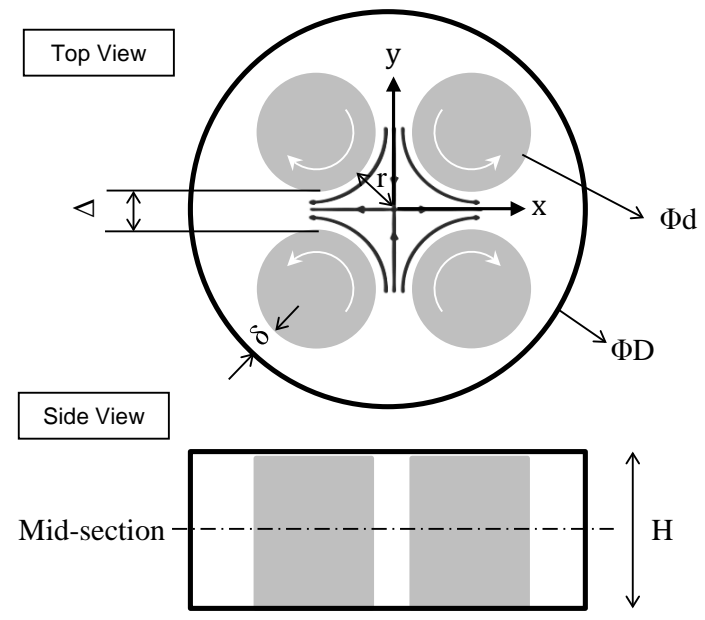

Figure 1: Schematic of a four-roll mill ( $d$ : diameter of rollers, $\Delta$ : gap (spacing) between rollers, $D$ : diameter of container, $\delta$ : gap between rollers and container, and $H$ : height of container.

A large number of researchers have measured and characterised the flow in four-roll mills [59 ] and have investigated the dynamics of single droplets in the stagnation point flow generated in four-roll mills [10-13].

Andreotti et al. [9] investigated the stability of the strain field in a four-roll mill. They showed that the pure straining flow becomes intrinsically unstable through a supercritical bifurcation to form an array of counter-rotating vortices aligned in the stretching direction. This was also confirmed by Philip and Cohen [14] using numerical simulation of an irrotational unbounded plane stagnation flow. It was found that the strain rate varies proportional to the rollers' frequency of the four-roll mill $\left(f_{4 r m}=\right.$ $2 \pi \Omega$ ), where $\Omega$ is the angular speed of the rollers [9]. In particular, the velocity gradient (strain rate) in the central region to scale as $f_{4 r m}$ :

$$
\frac{d u}{d x}=-\frac{d v}{d y}=\gamma=\kappa f_{4 r m}
$$

The proportionally coefficients were $\kappa=1.4$ for a mixture of $10 \%$ glycerol in water and $\kappa=2.7$ for the $99 \%$ glycerol [9]. These values were approximately a quarter of that $(\kappa=5.1)$ found by Taylor [3]. However, there is no report of coefficients of proportionality for water in previous works, as water is more susceptible to instability due to its reduced viscosity compared to other fluids, such as glycerol. Furthermore, it appears that $\kappa$ varies with the fluid medium as well as with the design parameters of the four-roll mill.

Pure extensional flow becomes intrinsically unstable through a supercritical bifurcation to form an array of counter-rotating vortices aligned in the stretching direction. The Reynolds number in a four-roll mill can be defined as [9]:

$$
R e_{\gamma}=\frac{\gamma \Delta^{2}}{\nu}
$$

where $\Delta$ is the spacing between four-roll mill rollers, $\gamma$ is the strain rate and $\nu$ is the kinematic viscosity. The critical Reynolds number is $R e_{\gamma, c r} \approx 17$ (for a four-roll mill with $H / d=2.7$ ).

Due to the existence of the moving elements in a four-roll mill, manufacturing a micron-sized fourroll mill is most challenging. Consequently, producing a steady hyperbolic flow at high rotational speed and high strain rates is difficult. This restriction stems from the growing Dean vortices from the top and bottom of a four-roll mill which make the extensional flow unstable $[9,15]$. Moreover, due to the low critical Reynolds number in a four-roll mill, it is necessary to use highly viscous fluid such as glycerol [7, 9]. Another potential issue caused by the low critical Reynolds number in a four-roll mill is the limitation in increasing the flow strain 
rate, as the strain rate is directly proportional to Reynolds number. The maximum strain rate that has been reached in a four-roll mill was reported to be about $7 \mathrm{~s}^{-1}$ [9]. In hindsight, these facts might appear to limit studying the dynamics of micronsized objects. We note that all the previous works used highly viscous fluids and large scale four-roll mills, where the gap between the rollers was $\mathrm{O}(10$ $\mathrm{mm}$ ) and higher.

\subsection{Cross-slot microfluidic device}

Another device that produces the same type of extensional stagnation point flow is a cross-slot microfluidic device, which can be used to study the deformation and break-up of micron-sized objects. A cross-slot type microfluidic device or simply a crossslot microchannel, consists of two micron-sized inlet channels with two converging streams and two outlet channels with two diverging streams. Both outlet channels (extensional axis) are set out perpendicular to the inlet channels (compressional axis) in a horizontal plane, schematically shown in figure 2 . At the intersection of the cross-junction, where the two laminar flows meet, a two-dimensional straining flow with a zero-velocity (stagnation) point is generated. The flow generated at the intersection of the cross-slot is similar to that of the Taylor's four-roll mill [3] with hyperbolic streamlines and the velocity field given by equation 1 .

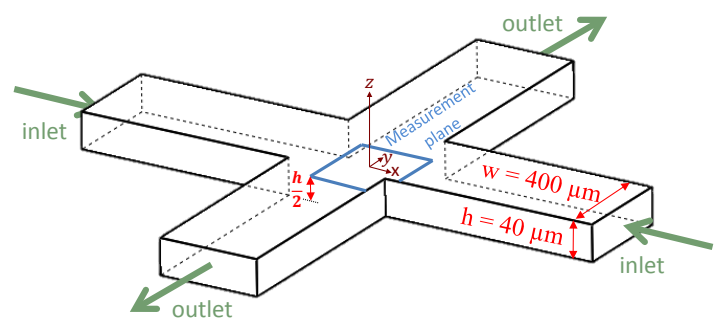

Figure 2: Three-dimensional schematic of the cross-slot channel. The dimensions represent the height and the width of the cross-slot microchannel used in this work.

Similar to four-roll mills, cross-slot microfluidic devices have been used for the characterisation and observation of deformation and breakup of bubbles and droplets [16]. However, they have also been used for confining micron-size beads [4, 1719], trapping a motile bacterium [20], stretching a DNA polymer strain [4, 21-23] and for phenotyp- ing of cancerous cells $[24,25]$ under pure straining flows. However, no systematic experimental measurements to characterise the flow in cross slot microchannels exist.

Cross-slot microchannels emerged with the advent of MEM's technology. They were first used to trap micron-sized objects such as DNA molecules [26]. However, this was limited to short-term confinement of the target as the stagnation point in this flow is a semi-equilibrium stable point. Over the last several years, using an image-based active control system, long-term trapping of a single micron-sized object became possible. Schroeder and coworkers $[4,17-20,23,27,28]$ developed an image-based micro-hydrodynamic trap in the straining flow of a cross-slot microchannel for highresolution confinement of single micron-sized objects. In all of the studies mentioned, the experiments were conducted at low strain rates (up to 1 $\mathrm{s}^{-1}$ ), as the aim of their experiments was to immobilise a target, such as fluorescent beads, single cells or stretching soft and very flexible DNA molecules.

Recently, cell phenotyping based on cell deformability using cross-slot microchannels has become popular [24, 25, 29, 30]. Gossett et al. [24] and Henry et al. [25] applied extremely high strain rates (about $10^{5} \mathrm{~s}^{-1}$ ) for a high throughput of 2000 cell/s. However, it is very likely that their crosschannels did not provide uniform strain rate flow with the presence of targets, as the size of the targets were relatively large compared to the width of the channels. Moreover, the residence time of the sample was $\mathrm{O}\left(10^{-5} \mathrm{~s}\right)$, which is not suitable for exposing the objects to a straining flow over a long period of time.

Regarding the velocity measurements at the cross-slot junction, some researchers obtained the velocity field by measuring the velocity of a few tracked particles and substituting their velocity in the velocity equations (equation 1) of the pure straining flow [4]. Others conducted micro-PIV measurements to measure the more accurate velocity field and the strain rate $[31,32]$. Some numerically simulated the flow in the cross junction using commercial software [33]. However, none of the investigations mentioned above used micro-PIV to fully characterise the flow in the cross-slot re- 
gion for a wide range of flow rate and accurately compare the flow with a pure straining flow by removing the bias error from the camera misalignment, and also investigating the small fluctuations of the stagnation point. Using a numerical simulation, Dylla-spears et al. [33] showed that the strain rate changes less than $5 \%$ within a radius of $w / 16$ in the vicinity of the stagnation point, where $w$ is the width of the channel. Pathak and Hudson [31] conducted micro-PIV to measure the velocity field of the flow of a non-Newtonian fluid and reported the strain rate changes less than $5 \%$ within a radius of $w / 4$.

\subsection{Present study}

The problem which motivated us to characterise straining flows was the cyanobacterium Anabaena circinalis $(10-300 \mu \mathrm{m}$ filamentous waterborne microorganisms), which is the cause of significant water contamination issues worldwide. Specifically, we are interested in the potential mechanical damage to cyanobacteria that may be caused by the strain rate of the flow [34-37]. The previous studies on the effect of strain rate on cyanobacteria were carried out in turbulent flows [34, 37, 38], from which only anecdotal evidence and sometimes opposite trends of suspected mechanical damage were reported (over a strain rate range of 2 and $\left.18 \mathrm{~s}^{-1}\right)$. Hence, our strategy here is to apply a spatio-temporally uniform high strain rate in a fluidic straining device using a stagnation point flow to characterise the physical strength of these species. There are also other studies, such as Lee et al. [39], who used an extensional flow, however without a stagnation point, consequently these devices cannot be used for confining objects. Furthermore, they are not capable of producing zero velocity point flow (pure extensional), which is our main interest. No such extensional flow can provide long-term confinement in a fixed location, which is suitable for our purpose.

In this paper, however, we only focus on the design, velocity and strain rate characterisation of micron-sized and miniature devices to produce extensional flows with high strain rate. Here, we report micro-PIV measurements in a cross-slot mi- crochannel and a miniature four-roll mill to obtain the velocity field and calculate the strain rate in both devices. The final objectives of these experiments are to calibrate the strain rate versus flow rate and also to determine the extent of the spatially uniform strain rate region, thereby studying the possibility of conducting experiments to investigate the effect of uniform and high enough strain rates on cyanobacteria. Furthermore, this feasibility study can be generalised for applying high strain rates to other micron-sized objects. No such experiment has been previously carried out to fully characterise the straining flow in a cross-slot microchannel and at the same time, to compare with a miniature four-roll mill with water as the medium. For this we manufactured a cross-slot micro channel with the width of $400 \mu \mathrm{m}$, and a four-roll mill with spacing between rollers of $500 \mu \mathrm{m}$, which is smaller than ever done previously.

\section{Four-roll mill: Experiments}

Experiments in water are crucial in this feasibility study since the waterborne microorganisms would not survive in a medium other than water. Previous studies used large size four-roll mills with highly viscous fluids instead of water. However, we require a miniature four-roll mill (almost 10 times smaller gap between the rollers) and with water as the medium. A miniature four-roll was designed and manufactured and a photograph of the final prototype is shown as an inset image in figure 3. The four-roll mill was designed and manufactured so that the effect of the size of the spacing between rollers alongside the size of the gap between rollers and container on the strain rate could be investigated. Four small glass windows (inside the black plastic frame in the inset image of figure 3) were made on the cylindrical wall of the container to provide optical access from the sides of the four-roll mill. A list of different configurations of the four-roll mill manufactured is presented in table 1. These dimensions were chosen based on the critical Reynolds number $\left(R e_{\gamma, c r} \approx 17\right)$ in fourroll mills. Assuming a strain rate of about $10 \mathrm{~s}^{-1}$ and considering the kinematic viscosity of water 
$\left(\nu \approx 10^{-6} \mathrm{~m} / \mathrm{s}^{2}\right)$ resulted in the rollers spacing of 0.5 to $1 \mathrm{~mm}$. To obtain the best approximation of a hyperbolic flow equation we obtain from figure 1 [9]:

$$
\left(\frac{d}{2}+r\right)^{2} \approx 2\left(\frac{d}{2}+\frac{\Delta}{2}\right)^{2}
$$

where $(d / 2+r)$ is the distance between the roller's axis and the centre of the four-roll mill. The dimensions listed in table 1 were chosen in order to satisfy equation 4 .

Table 1: Dimensions of the miniature four-roll based on the parameters shown in figure 1.

\begin{tabular}{cccccc}
\hline \hline Dimensions $(\mathrm{mm})$ & $d$ & $D$ & $\Delta$ & $\delta$ & $H$ \\
\hline Case $A$ & 8 & 22.45 & 1.5 & 0.5 & 16 \\
Case $B$ & 9 & 22.45 & 0.5 & 0.5 & 16 \\
Case $C$ & 9 & 28.45 & 0.5 & 3 & 16
\end{tabular}

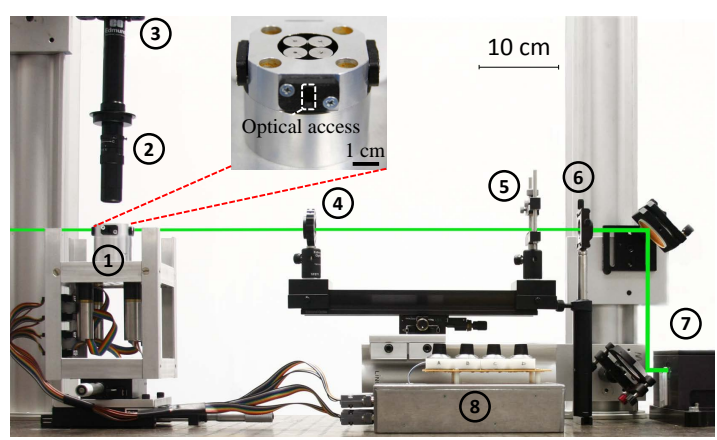

Figure 3: The planar-PIV setup to measure the velocity in the miniature four-roll mill. (1) Four-roll mill. (2) Zoom lens. (3) PIV camera. (4) Spherical lens. (6) Aperture. (7) Laser. (8) Controller.

Planar-PIV was used to measure the velocity field in the mid-section (shown in the bottom panel of figure 1) of the four-roll mill. The working fluid (MiliQ water) was seeded with one micron diameter red fluorescent polystyrene aqueous microspheres (ThermoFisher Scientific). A high speed camera with a $15.5 \times 12.3 \mathrm{~mm}^{2}$ CMOS sensor $(1280 \times 1024,10$ bits, IDT Y3-Classic), that is able to capture back-to-back images at $1000 \mathrm{fps}$ at full resolution, was coupled with a C-mount 2.5-10× $\mathrm{VZM}^{\mathrm{TM}}$ 1000i zoom lens to record the particle images. The minimum exposure of the camera and the pixel spacing were $1 \mu$ s and $12 \mu \mathrm{m}$, respectively. A $532 \mathrm{~nm}$ Nd:YAG double-pulsed laser (120 mJ per 5 ns pulse, CFR - BigSky Laser Series) capable of double pulsing at $10 \mathrm{~Hz}$ was used to illuminate the flow. A cylindrical and a spherical lens as well as an aperture (to partially block the light beam) were used to form $150 \mu \mathrm{m}$ light sheet passing through the centre of the mid-section of the four-roll mill. Relevant PIV parameters are listed in table 2, and the experimental setup and its components are shown in figure 3. The four-roll mill was operated at two different angular speeds of 75 and $150 \mathrm{rpm}$. At higher angular speeds, for the different cases listed in table 1 , the flow became unstable.

Table 2: PIV and experimental parameters

\begin{tabular}{ll}
\hline \hline Image size $(\mathrm{px})$ & $1280 \times 1024$ \\
Lens magnification & $10 \times$ \\
Optical magnification $(\mu \mathrm{m} / \mathrm{px})$ & $\approx 1.5$ \\
Depth of focus $(\mu \mathrm{m})$ & 50 \\
Laser light sheet thickness $(\mu \mathrm{m})$ & $\approx 150$ \\
\hline Field of view $(\mathrm{mm} \times \mathrm{mm})$ & $1 \times 1$ \\
Interrogation window size $(\mathrm{px})$ & $32 \times 32$ \\
Interrogation windows overlap & $50 \%$ \\
\hline Red fluorescent polystyrene microspheres \\
Particles diameter (px) & $\approx 1 \mu \mathrm{m}$ \\
Flow media & MiliQ water \\
& $99 \%$ Glycerol \\
\hline
\end{tabular}

For PIV, a multipass algorithm with adaptive central difference interrogation (CDI) offsetting was employed (the parameters are listed in table 2). The multipass technique was combined with a window deformation algorithm $[40,41]$ to reduce the random error caused by deformation of the measured flow.

\section{Cross-slot microchannel: Experiments}

The complete fabrication protocol of the microchannels using soft-lithography has been detailed in Akabridoust et al. [42]. In short, using this soft-lithography protocol, a microfluidic device with features shown in figure 4 and originally designed by Johnson-Chavarria [20] was fabricated. A negative photo-lithographic mask was manufactured using maskless lithography. This was followed by the fabrication of a master mould using contact-lithography. The master mould consists of the SU-8 cured onto a silicon substrate. By casting polydimethylsiloxane (PDMS), Sylgard 184 with a 
base:crosslinker ratio of $1: 10$, against the master moulds the microchannel features on the mould were replicated on the PDMS slab. Finally, the PDMS replica was bonded to a glass slide to form a sealed microfluidic device using oxygen plasmacleaning. The height and width of the channel are 40 and $400 \mu \mathrm{m}$, respectively (an aspect ratio of $\alpha=h / w=0.1)$. As shown in figure 4 the sample injection port is where the micron-sized objects could be injected into the flow.

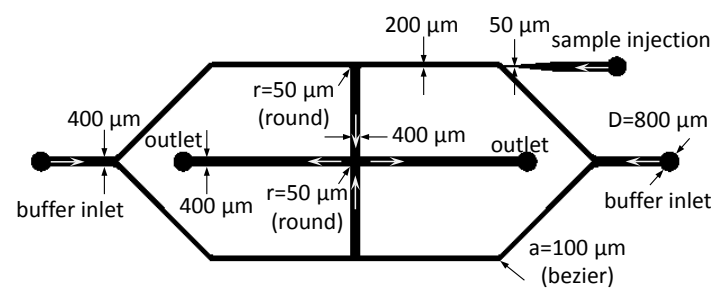

Figure 4: Design of cross-slot microchannel used in present work, and originally designed by JohnsonChavarria [20].

A high speed CMOS sensor camera $(2000 \times 2000$ pixels, 12 bits, PCO.dimax HS4), that is able to capture back-to-back images at $2277 \mathrm{fps}$ at full resolution, was coupled with an inverted TI-U Eclipse Nikon microscope side port to record the particle images. The microscope was equipped with a CFI S Plan Fluor ELWD $20 \times(\mathrm{NA}=0.45)$ objective, ideal for fluorescence imaging. Velocity measurements at the mid-plane of the cross-slot microchannel was carried out using micro-PIV. To illuminate the flow a $532 \mathrm{~nm} \mathrm{Nd:YAG} \mathrm{double-pulsed} \mathrm{laser} \mathrm{(200}$ mJ per 5 ns pulse, EverGreen - BigSky Laser Series) capable of double pulsing at $15 \mathrm{~Hz}$ was used to illuminate the flow.

The flow was seeded with one micron diameter red fluorescent polystyrene aqueous microspheres (ThermoFisher Scientific). In order to drive the fluid into the microchannel, a gas-tight glass syringe (1000 $\mu \mathrm{L}$, Hamilton) and a programmable syringe pump (Harvard PHD ULTRA) were used. Luer lock adaptors and 24 gauge bent metal tubing were used to connect the perfluoroalkoxy (PFA) tubes to the syringe and access ports of the microfluidic device via PFA tubes (0.020 inch inner diameter (ID), 1/16 inch outer diameter (OD), IDEX), respectively. A manual micro shut-off valve (1/16 inch OD tubing Upchurch) was used to block the injection port. The same metal tubing and PFA tubes were used to establish the connection between the microfluidic device and the drain reservoir. To maintain a constant pressure drop, the outlet tubes were of equal length and both were submerged into a sample container. The tubes were pre-filled to reduce the quantity of bubbles in the flow.

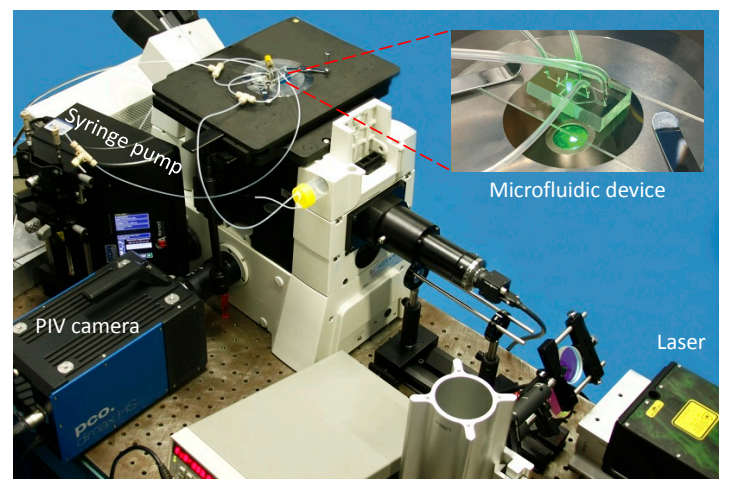

Figure 5: Photograph of the micro-PIV and experimental setup. Inset figure shows the fabricated microfluidic device mounted onto the microscope stage and its connection to the syringes and pump.

The microfluidic device was horizontally mounted and firmly clamped onto the inverted microscope stage (shown in figure 5). The connection between the microfluidic device and the $1000 \mu \mathrm{L}$ syringe was established, and using the shut-off valve, the injection port was blocked. The syringe pump delivered the fluid through the inlet ports at a high flow rate $(4000-5000 \mu \mathrm{L} / \mathrm{hr})$. Delivering the fluid to the microchannels at high flow rates assists in removing the bubbles from the channels. Then the flow rate was gradually decreased to $50 \mu \mathrm{L} / \mathrm{hr}$. For each flow rate, 3600 pairs of PIV images were recorded for four minutes $(15 \mathrm{~Hz})$. For each measurement, different time intervals for laser pulses were chosen, varying from $12 \mathrm{~ms}$ to 60 $\mu$ s for the flow rates between 50 to $3000 \mu \mathrm{L} / \mathrm{hr}$.

A schematic of the micro-PIV setup is shown in figure 6. The relevant micro-PIV parameters are listed in table 3. PIV parameters are listed in table 3 and processing methods are provided in appendix A. We used a combination of the common averaging methods instead of a single ensembleaveraging method, to study the semi time-resolved velocity field and measure the possible fluctuation of the stagnation point and simultaneously avoid the problem of micro-PIV low image density. 


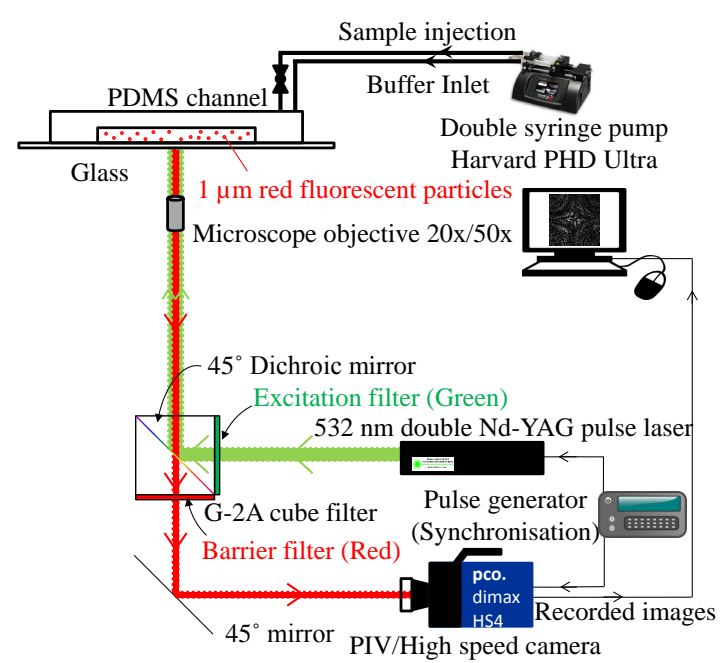

Figure 6: Schematic of the micro-PIV setup.

The depth of correlation $\left(\delta z_{c}\right)$ is calculated using [43]:

$\delta z_{c}=2\left[\frac{1-\sqrt{\epsilon_{t h}}}{\sqrt{\epsilon_{t h}}}\left(\frac{d_{p}^{2}}{4 \mathrm{NA}^{2}}+\frac{1.49(\mathrm{M}+1)^{2} \lambda_{0}}{\mathrm{M}^{2} \mathrm{NA}^{4}}\right)\right]^{0.5}$

where $\mathrm{M}=20 \times$ and NA $=0.45$ are the magnification and numerical aperture, respectively, of the objective lens. Parameter $\epsilon_{t h}$ is a threshold that determines whether an image of the particle can contribute the correlation function (typically equal to 0.01 [43]). As such, the depth of correlation was $7.8 \mu \mathrm{m}$, which is suitable for this feasibility study as the thickness of the target objects (diameter of the cell of a Anabaena circinalis filaments) is between 5 to 10 microns.

Table 3: Micro-PIV and experimental parameters

\begin{tabular}{lc}
\hline \hline Objective magnification $(\mathrm{M})$ & $20 \times$ \\
Numerical aperture $(\mathrm{NA})$ & 0.45 \\
\hline Image size & $800 \times 800(\mathrm{px})$ \\
Optical magnification & $\approx 0.55(\mu \mathrm{m} / \mathrm{px})$ \\
Depth of field & $\approx 2.5(\mu \mathrm{m})$ \\
Depth of correlation $\left(\delta z_{c}\right)$ & $\approx 7.8(\mu \mathrm{m})$ \\
Field of view & $360 \times 360\left(\mu \mathrm{m}^{2}\right)$ \\
\hline Number of overlaid images & 6 \\
Frequency & $5(\mathrm{~Hz})$ \\
Interrogation window size & $32 \times 32(\mathrm{px})$ \\
Overlap & $50 \%$ \\
Spatial resolution & $\approx 9(\mu \mathrm{m})$ \\
\hline Red fluorescent polystyrene microspheres \\
Particle diameter $\left(d_{p}\right)$ & $\approx 1(\mu \mathrm{m})$ \\
Flow medium & Distilled water \\
\hline
\end{tabular}

Due to the relatively high speed flow in this study (order of $1000 \mu \mathrm{m} / \mathrm{s}$ ), the maximum relative error [44] associated with the Brownian motion was insignificant (maximum $0.25 \%$ ). This amount is considerably lower than that of other micro-PIV experiments, which is usually about $10 \%$ [44-46]. In our experiments the effective diameter of the particle image $[44,45]$ was $d_{e}=28.8 \mu \mathrm{m}$ and when it is projected back to the flow it is $1.44 \mu \mathrm{m}$. Therefore, the micro-PIV measurement uncertainty [47] was $144 \mathrm{~nm}$.

\section{Four-roll mill: Results and discussions}

Figure 7 shows an example of the velocity vector map in the $1 \mathrm{~mm} \times 1 \mathrm{~mm}$ central region of the four${ }_{.5}$ roll mill (shown in figure 1 ) at the rollers' angular speed of $\Omega=70 \mathrm{rpm}$.

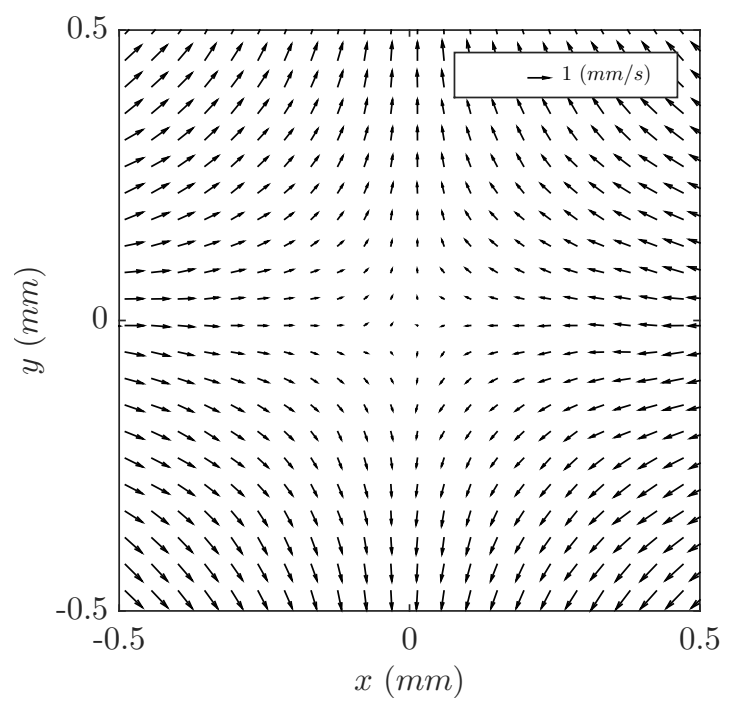

Figure 7: Velocity vector map in the $1 \mathrm{~mm} \times 1 \mathrm{~mm}$ central region of the four-roll mill (shown in figure 1) at the rollers' angular speed of $\Omega=75 \mathrm{rpm}$.

To calculate the strain rate, the gradients of velocity map $\left(\frac{\partial u}{\partial x}\right)$ and $\left(\frac{\partial v}{\partial y}\right)$ were calculated by fitting a Savitzky-Golay differentiation filter on a set of $5 \times 5$ velocity data points to a third-order polynomial in the least-squares sense. Strain rate was calculated by:

$$
\gamma=\frac{1}{2}\left(\frac{\partial u}{\partial x}+\frac{\partial v}{\partial y}\right)
$$

In order to quantitatively compare the mea- 
sured velocity field and the uniformity of the strain rate to an ideal hyperbolic flow, equations:

$$
u=\gamma_{e q}\left(x-X_{o}\right), \quad v=-\gamma_{e q}\left(y-Y_{o}\right),
$$

that present a linear velocity field with an unknown centre of $\left(X_{o}, Y_{o}\right)$ and unknown $\gamma_{e q}$ (an equivalent strain rate that is a constant strain rate for a given flow rate) were fitted to the velocity field. Non-linear equations were solved using the NewtonRaphson method, and the position of centre $\left(X_{o}\right.$, $\left.Y_{o}\right)$ and the equivalent strain rate $\left(\gamma_{e q}\right)$ were determined.

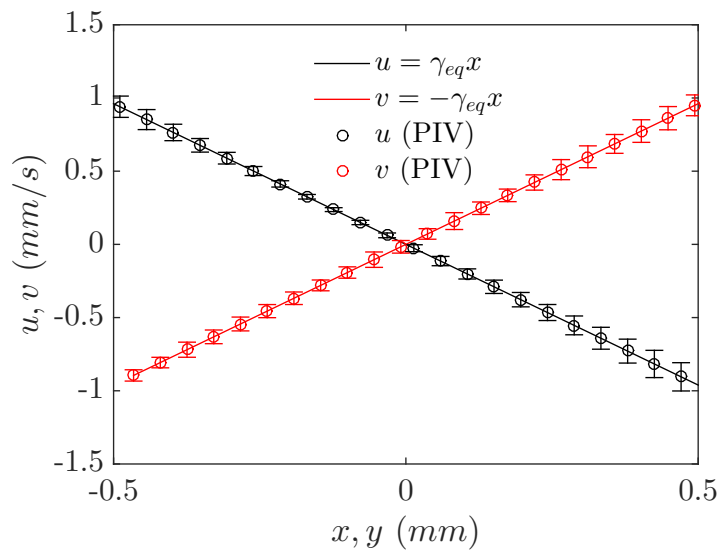

Figure 8: The solid lines represent the least square fits to the ensemble-averaged velocity and the error bars correspond to twice the standard deviation of the $95 \%$ confidence level.

Figure 8 shows the deviation of the flow in the four-roll mill from a pure extensional flow (equation 1). The black and red solid lines in figure 8 show the least-square fits of the pure extensional flow equations to the values of $u$ and $v$, respectively. The error bars of the $u$-velocity and $v$-velocity data show twice the standard deviation in the respective directions. With the increase in the distance from the stagnation point, an increase in deviation for both velocity components from the linear profile can be seen. This deviation is rooted in the effect of the rollers disrupting the ideal extensional flow. The deviation from a uniform straining flow in the central region $1 \mathrm{~mm} \times 1 \mathrm{~mm}$ was less than $10 \%$. In this region the relation $\frac{d u}{d x}=\frac{d v}{d y}=\gamma$ is held within an accuracy of $10 \%$.

Figure 9 illustrates fluctuations of the stagnation point $\left(X_{o}, Y_{o}\right)$ for the roller angular speed of $\Omega=75 \mathrm{rpm}$, and figure 10 shows the fluctuation of $\gamma_{e q}$ for both angular speeds of $\Omega=75$ and $150 \mathrm{rpm}$. Frequencies (computed using Fast Fourier Transform (FFT)) and the standard deviations of the stagnation point position and the strain rate signals for all the four-roll mill cases are listed in table 4.

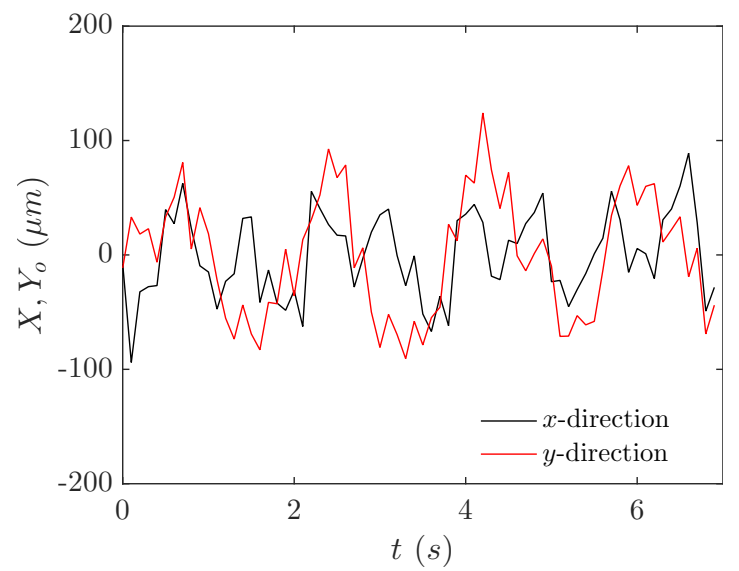

Figure 9: Stagnation point fluctuations in $x$ and $y$ directions at rollers' angular speed of $\Omega=75 \mathrm{rpm}$.

The important observation is that when the frequencies are converted to rpm, the frequencies of the signals (for both cases of water and glycerol $99 \%$ as the medium) are very close to the angular speeds of the rollers. This strongly suggests that the variation in the position of the roller axis is influencing the variation of the stagnation point. The standard deviation of stagnation point position is the order of magnitude of 10 microns. The maximum strain rate achieved is found to be about 3 and $6 \mathrm{~s}^{-1}$ for laminar flows of water and glycerol $99 \%$, respectively. In our miniature four-roll, it is possible to reach higher strain rate when using glycerol as the medium due to the low Reynolds number. However, as mentioned earlier, it is necessary for us to use water as the medium.

These fluctuations most likely stem from slight eccentric and unsynchronised rotations of the rollers. By monitoring the signal of the circuit of the rollers, it was found that each roller has $3 \%$ inconsistency in the magnitude of its angular speed. Whilst to quantify the possible eccentric rotation, the rotating rollers were imaged and the locations of some arbitrary points on the edges of the rollers were tracked.

To confirm that the fluctuations of the stagna- 
Table 4: Frequency and standard deviation of the strain rate and the positions of the stagnation point in the four-roll mill for different dimensions listed in table 1.

\begin{tabular}{|c|c|c|c|c|c|c|c|c|}
\hline & & \multicolumn{4}{|c|}{ Stagnation point } & \multicolumn{3}{|c|}{ Strain rate } \\
\hline$\Omega(\mathrm{rpm})$ & $R e_{\gamma}$ & $\begin{array}{c}\sigma(\mu \mathrm{m}) \\
x \text {-direction }\end{array}$ & $\begin{array}{c}\sigma(\mu \mathrm{m}) \\
y \text {-direction }\end{array}$ & $\begin{array}{c}f_{\text {st }}(\mathrm{rpm}) \\
x \text {-direction }\end{array}$ & $\begin{array}{c}f_{s t}(\mathrm{rpm}) \\
y \text {-direction }\end{array}$ & $\gamma_{e q}\left(\mathrm{~s}^{-1}\right)$ & $\sigma_{\gamma}\left(\mathrm{s}^{-1}\right)$ & $f_{\gamma}(\mathrm{rpm})$ \\
\hline \multicolumn{9}{|c|}{ Water in four-roll mill case A } \\
\hline 75 & 5.40 & 51.9 & 52.3 & 72 & 70 & 2.4 & 0.054 & 70 \\
\hline 150 & 6.97 & 92.1 & 44.3 & 155 & 150 & 3.1 & 0.186 & 150 \\
\hline \multicolumn{9}{|c|}{ Glycerol $99 \%$ in four-roll mill case A } \\
\hline 75 & 0.011 & 25.8 & 34.7 & 72 & 70 & 3.2 & 0.048 & 72 \\
\hline 150 & 0.012 & 40.2 & 69.9 & 153 & 152 & 6.1 & 0.187 & 152 \\
\hline \multicolumn{9}{|c|}{ Water in four-roll mill case B } \\
\hline 75 & 5.39 & 18 & 52.3 & 70 & 75 & 5.09 & 0.072 & 72 \\
\hline 150 & 5.56 & 41.2 & 76.4 & 154 & 150 & 5.56 & 0.43 & 150 \\
\hline \multicolumn{9}{|c|}{ Water in four-roll mill case $\mathrm{C}$} \\
\hline 75 & 0.93 & 95.4 & 78.6 & 70 & 75 & 3.7 & 0.088 & 70 \\
\hline
\end{tabular}

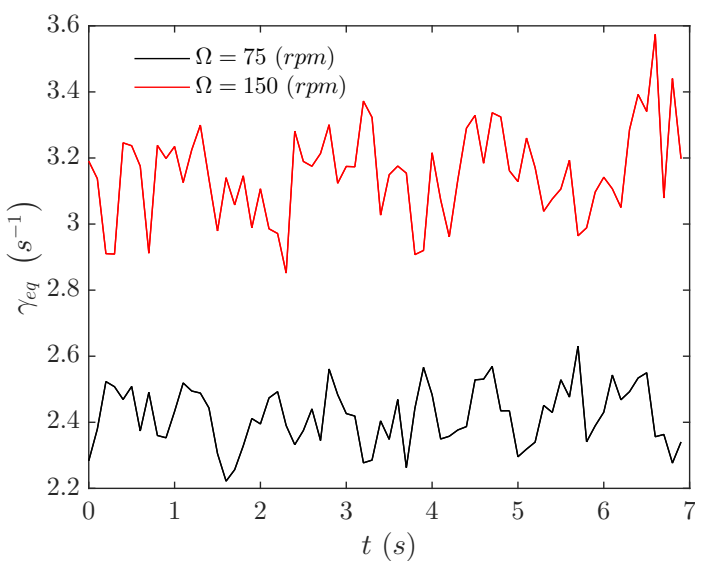

Figure 10: Fluctuation of the strain rate in the central region of $1 \mathrm{~mm} \times 1 \mathrm{~mm}$ at both rollers' angular speed of $\Omega=75$ and $150 \mathrm{rpm}$.

tion point are caused by the eccentric rollers rotation, we simulated the flow in the whole cell of the four-roll mill by a potential flow. Full details of the simulation are given in appendix B. Applying the $3 \%$ unsynchronisation (similar to the experiment) in the magnitude angular speed of the rollers and $50 \mu \mathrm{m}$ eccentric rotation of the four vortices of the potential flow the fluctuations in the position of the stagnation point were obtained and shown in figure 11. The standard deviation of the signal is close to the standard deviation of the fluctuations of the stagnation point measured using PIV. Therefore, it can be deduced that the movement of the stagnation point is caused by the eccentric rotation of the rollers. However, considering the small size of the four-roll mill, making more precise rollers is extremely challenging and becomes exorbitantly expensive with our current manufacturing capabilities.

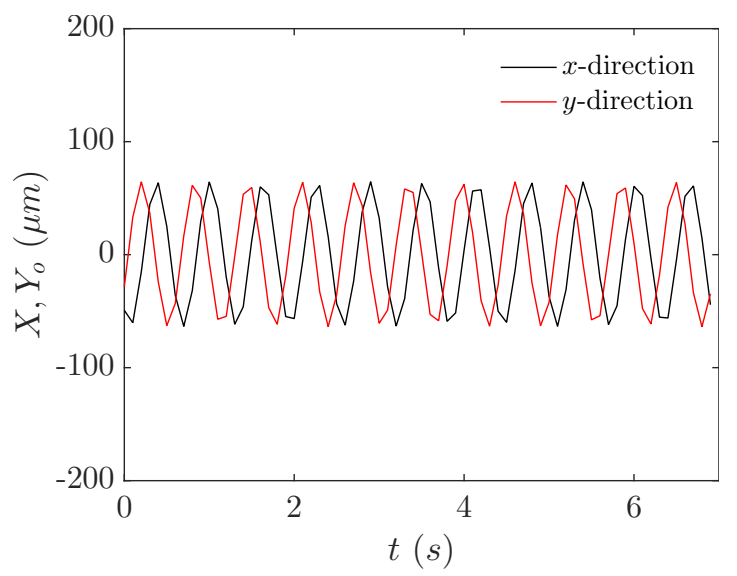

Figure 11: The fluctuations of the stagnation point in the simulated four-roll mill obtained from the potential flow simulation.

Finally the strain rates for all three different cases of dimensions listed in table 1 are shown in figure 12. The results for glycerol $99 \%$ agrees with Andreotti et al. [9], the same fluid was used and our four-roll mill has similar dimension ratios to their apparatus. Moreover, the figure shows that decreasing the gap between the rollers (in other words increasing the diameters of rollers) leads to an increase in the strain rate. This happened due to the increase in the linear velocity of the rollers and 


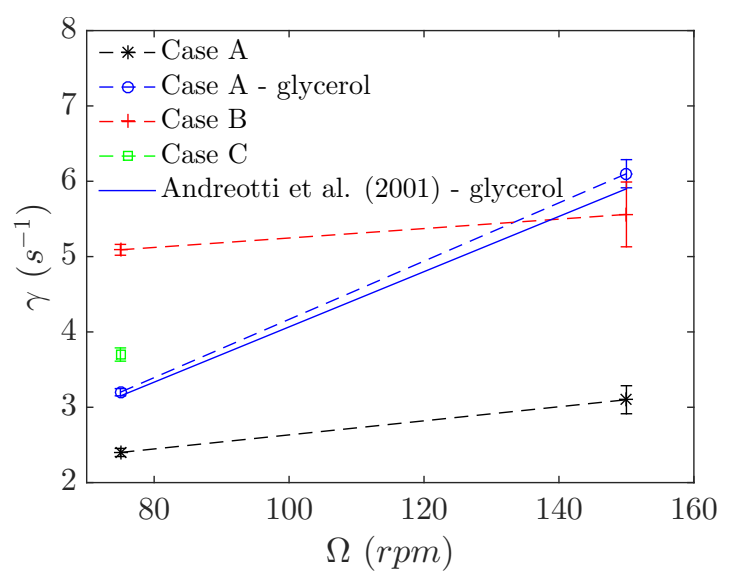

Figure 12: Strain rate in four-roll mill at different rollers' angular speed.

the resulting flow velocity and gradients. However, the proportionality coefficient is the same for both cases and is found to be $\kappa=0.06$. Additionally, the single data-point for the large container (Case C) shows a decrease in the strain rate compared to the smaller container (Case B).

\section{Cross-slot microchannel: Results and discussions}

Figure 13 shows an example of the ensembleaveraged velocity vector map and streamlines in the $300 \mu \mathrm{m} \times 300 \mu \mathrm{m}$ central region of the crossslot junction (shown in figure 2) at the flow rate of $75 \mu \mathrm{L} / \mathrm{hr}$. To qualitatively compare the flow to the pure straining flow, the velocity magnitude contour is depicted in figure 14. The circular regions in this figure confirm the existence of a linear velocity field, as pure straining flow has a circular velocity magnitude profile $\left(\sqrt{u^{2}+v^{2}}=\gamma \sqrt{x^{2}+y^{2}}\right)$.

Using the same calculation procedure for fourroll mill, $X_{o}, Y_{o}$ and $\gamma_{e q}$ were obtained from PIV velocity fields for each time instance. The results for time-averaged $\gamma_{e q}$ is shown in figure 15, where the black and red solid lines in this figure show the least-square fits to the ensemble-averaged values of $u$ and $v$, respectively. The error bars of the $u$-velocity and $v$-velocity data show twice the standard deviation of the $95 \%$ level along the $y$-axis (extensional axis) and $x$-axis (compressional axis), respectively. With the increase in distance from the stagnation point, an increase in deviation for both

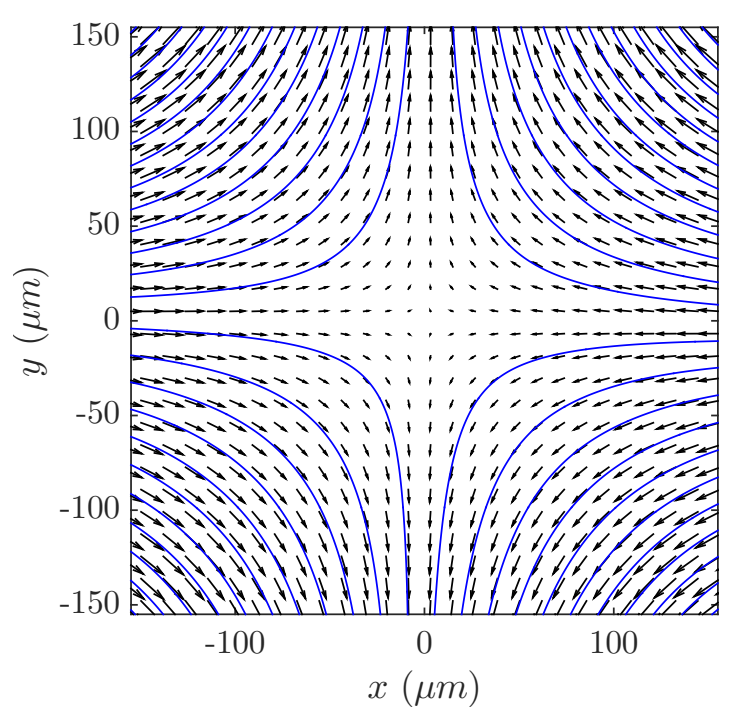

Figure 13: Ensemble-averaged velocity vectors map and streamlines in a $300 \mu \mathrm{m} \times 300 \mu \mathrm{m}$ central region of the cross-slot junction at the flow rate of $75 \mu \mathrm{L} / \mathrm{hr}$.

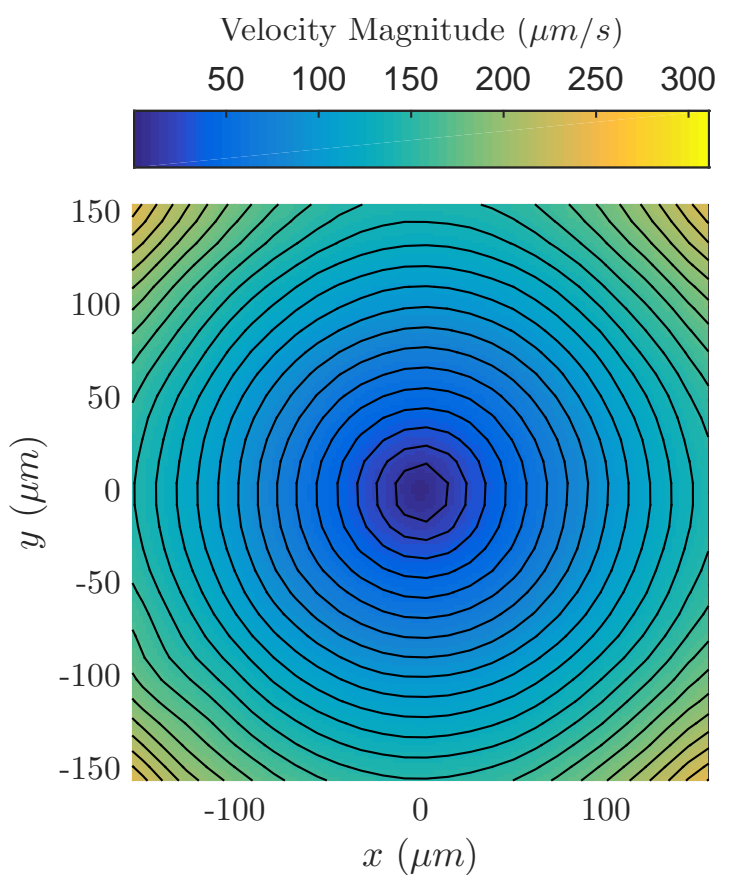

Figure 14: Ensemble-averaged velocity magnitude in a $300 \mu \mathrm{m} \times 300 \mu \mathrm{m}$ central region of the cross-slot junction at the flow rate of $75 \mu \mathrm{L} / \mathrm{hr}$. Circular contour lines are an indication of the linear stagnation point flow.

velocity components from the linear profile can be seen. This deviation might be rooted in the effect of the side walls.

More importantly, it is evident that the error bars are not symmetric around the $x$ and $y$-axes. This means bias errors exist which are not caused by the effect of the cross-slot walls, as the channel has a symmetrical geometry. We found that the bias error was caused by an angular misalignment 


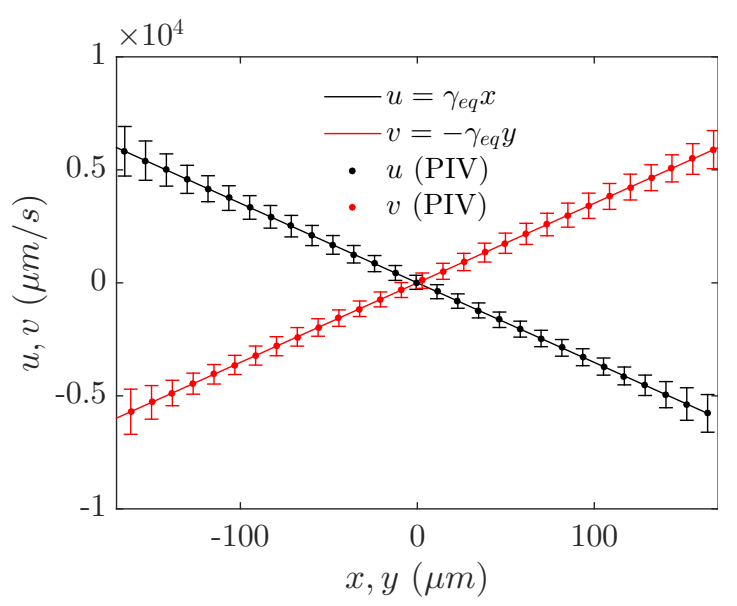

Figure 15: The solid lines represent the least-square fits to the ensemble-averaged velocity and the error bars correspond to twice the standard deviation of the $95 \%$ confidence level.

of the camera and the microchannel (with a maximum misalignment angle found later to be about $\left.1.5^{\circ}\right)$. To address this problem the whole velocity field was tilted around the intersection of these two lines. Therefore, the bias error in the deviation of the measured velocity from the ideal linear flow was removed from the data and the deviations reduced to half of their initial values. It can also be concluded that for all the flow rates, the deviation from a uniform straining flow in the central region $(-0.6 w<x, y<0.6 w)$ was less than $2 \%$. In other words, in this region the relation $\frac{d u}{d x}=\frac{d v}{d y}=\gamma$ is held with the accuracy of $2 \%$. The details of calculations are provided in appendix $\mathrm{C}$.

As previously mentioned, we obtained the time resolved velocity to investigate the possible variation of the stagnation point location with time, and compared this with our miniature four-roll mill. Figure 16 shows the fluctuation of the stagnation point location and $\gamma_{e q}$ for the case of flow rate $Q=700 \mu \mathrm{L} / \mathrm{hr}$. The standard deviation of the stagnation point variation in $x$ and $y$ directions are $0.50 \mu \mathrm{m}$ and $0.63 \mu \mathrm{m}$, respectively. Using the same method, it is confirmed that these values were less than one micron for all the flow rates, whereas those of the four-roll mill were higher than $30 \mu \mathrm{m}$. This result confirms the possibility of trapping and confining a target object larger than one micron at the zero-velocity point.

Note that there is an observable periodic behaviour in $\gamma_{e q}$ data (figure 16c), whereas no such

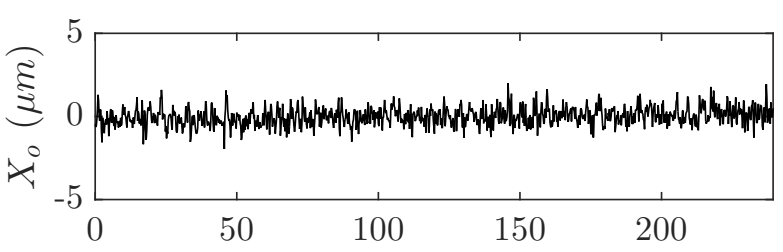

(a) Location of the stagnation point in $x$-direction.

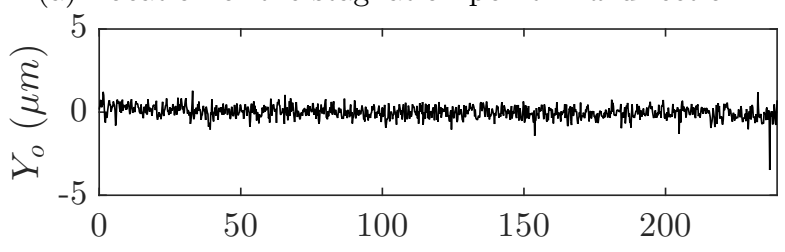

(b) Location of the stagnation point in $y$-direction.

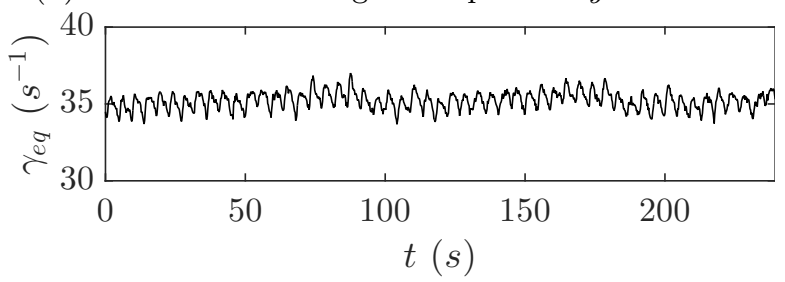

(c) Strain rate.

Figure 16: Fluctuation of the stagnation point and the strain rate over a four minute measurement. The variation of the stagnation point was less than $1 \mu \mathrm{m}$ for all the flow rates and the variation of the strain rate was less than $2 \%$.

periodic feature is observed in the location of the stagnation point (figures $16 \mathrm{a}$ and $16 \mathrm{~b}$ ). We suspect that the fluctuation in $\gamma_{e q}$ is due to the pumping of fluid and related to the flow rate. If this is true, we would expect the dominant frequency in strain rate $\left(f_{\gamma}\right)$ scales linearly with flow rate $(Q)$. The FFT of $\gamma_{e q}$ in figure $16 \mathrm{c}$ shows $f_{\gamma} \approx 0.22 \mathrm{~Hz}$. After analysing time-series for all flow rates $(\mathrm{Q})$, we found that indeed $f_{\gamma}$ has a linear relationship to $Q$ $\left(f_{\gamma}=1.13 Q\right)$. In any case, the amplitude of this frequency is so small that it hardly affects the uniformity of the flow strain rate (The standard deviation of $2 \%$ ). However, $X_{o}$ and $Y_{o}$ did not show this trend, most likely because the inlets are symmetric and the oscillation cancels out.

To provide the possibility of the investigation into uniformity of strain rate for all the flow rates together, the non-dimensional parameter $\gamma^{*}$ is defined as:

$$
\gamma^{*}=\frac{\langle\gamma\rangle-\left\langle\gamma_{e q}\right\rangle}{\bar{V} /(2 w)},
$$

where $\bar{V}=Q /(2 w h)$ is the mean velocity, $\langle\gamma\rangle$ is the time-averaged strain rate and is a function of flow 


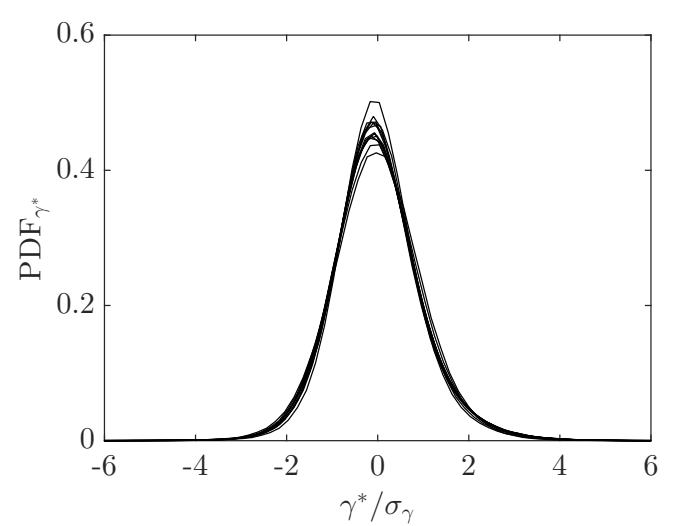

Figure 17: PDF of non-dimensional parameter $\gamma^{*}$ for various flow rates, showing the same deviation in the central region of the cross junction.

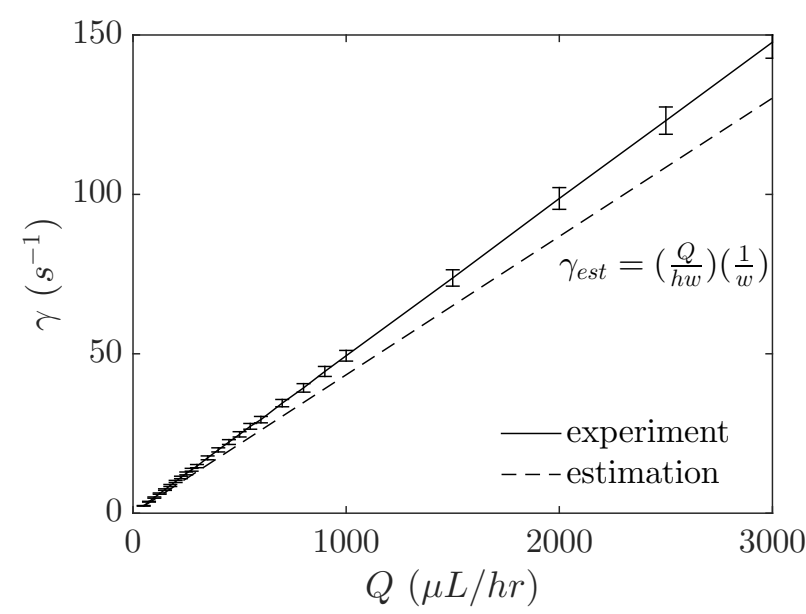

Figure 18: Strain rate versus flow rate at the cross junction, the error bars represent twice the standard deviation of the $95 \%$ confidence level of the measured data and the dash line shows the crude estimation of the strain rate.

rate (Q). While, $\left\langle\gamma_{e q}\right\rangle$ is the time-averaged of $\gamma_{e q}$ and is a function of $x, y$ and $Q$. Probability Density Functions (PDF) of the non-dimensional strain rate for all of the flow rates are shown in figure 17 . The figure shows the same standard deviation in the central region for all flow rates. The relative standard deviation was nominally $2 \%$.

Finally the strain rate of the velocity field at a central region of the cross-slot microchannel $(-0.6 w<x, y<0.6 w)$ is plotted in figure 18. The error bars represent twice the standard deviation of the strain rate over this region. A common practice [32] is to relate the strain rate and flow rate by a linear relationship. In the present study, based on least-square fit we found:

$$
\gamma=0.0493 Q+0.0426
$$

where $Q$ is in $(\mu \mathrm{L} / \mathrm{hr})$ and $\gamma$ is in $\left(\mathrm{s}^{-1}\right)$. The strain rate in the cross junction can be crudely estimated using [16]:

$$
\gamma_{e s t}=\left(\frac{Q}{h w}\right)\left(\frac{1}{w}\right),
$$

which is the average inlet velocity divided by half of the width of the channel, where velocity reaches from its maximum value to zero. Therefore, due to the effect of the wall, this formula underestimates the strain rate. Also by increasing $Q$ a systematic departure from the experimental data was observed. This estimation is shown in figure 18 along with the measured strain rates.

For a more systematic analysis of the dependence of $\gamma_{e q}$ on the experimental parameters, we propose a non-dimensional analysis. Therein, $\gamma_{e q}$ is assumed to be a function of the flow rate $(Q)$, the geometry parameters ( $h$ and $w$ ), and fluid properties - density $(\rho)$ and viscosity $(\mu)$. The dimensional analysis would suggest:

$$
\frac{\gamma_{e q}}{\left(\frac{Q}{h w}\right)\left(\frac{1}{w}\right)}=\text { function }\left(R e_{D_{h}}, \alpha\right)
$$

where $R e_{D_{h}}$ is Reynolds number $\left(R e_{D_{h}}=\right.$ $\left.\rho \bar{V} D_{h} / \mu\right), \quad D_{h}$ is hydraulic diameter $\left(D_{h}=\right.$ $h w /(2 h+2 w))$ and $\alpha=h / w$. For our study, we have only a single $\alpha=0.1$, and figure 19 shows the dependence of $\gamma_{e q} / \gamma_{e s t}$ on $R e_{D_{h}}$. However, $\gamma_{e q} / \gamma_{e s t}$ turns out to be a constant within the measurement errors, and it is found that $\gamma_{e q} / \gamma_{e s t}=1.13 \pm 0.02$. In future it would be worthwhile to consider other aspect ratios $(\alpha)$. In any case, the non-dimensional analysis provides a framework to fully characterise the strain rate at the cross-slot junction.

It is seen that strain rates of up to $142 \mathrm{~s}^{-1}$ could be reached in this microfluidic device, whereas 6 $\mathrm{s}^{-1}$ is the highest reported strain rate in conventional four-roll mills [9] and in our miniature four roll-mill. Therefore, not only does the cross-slot microchannel enable the study of micron-sized objects in a uniform straining flow, but it also provides the possibility of applying a high strain rate to micronscaled objects.

It should be noted that due to the no-slip con- 


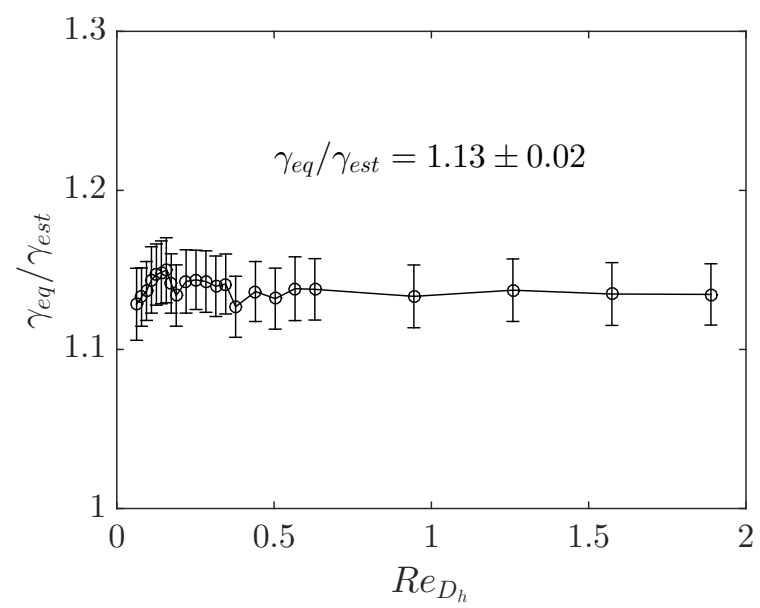

Figure 19: Dependence of $\gamma_{e q} / \gamma_{e s t}$ on $R e_{D_{h}} \cdot \gamma_{e q} / \gamma_{e s t}$ turns out to be a constant within the measurement errors, and it is found $\gamma_{e q} / \gamma_{e s t}=1.13 \pm 0.02$.

dition at the top and bottom walls of the crossslot channel, the flow is only approximately twodimensional and the strain rate varies at different $z$ planes. The strain rates reported in this study correspond to the average strain rate along $\delta z_{c} \approx 7 \mu \mathrm{m}$ (17\% of the channel height) over the mid-section of the channel, which is close to the thickness of $A$. circinalis filaments (about 5 to $10 \mu \mathrm{m}$ ). Therefore, when a $A$. circinalis filament is held at the stagnation point in the mid-section of the channel, it will be exposed to a flow with strain rates that are measured here.

\section{Summary and conclusions}

A cross-slot microchannel and a miniature four-roll mill (with the gap 10 times smaller than previous works) were manufactured to study the possibility of investigating the effect of strain rate in flow on waterborne microorganisms.

Using a combination of the common averaging methods instead of a single ensemble-averaging method, enabled us to study the semi time-resolved velocity field and measure the possible fluctuation of the stagnation point and simultaneously avoid the problem of micro-PIV low image density.

The cross-slot microchannel was found to be a better experimental platform than a miniature four-roll mill for the purpose of confining micronsized objects in a controlled extensional flow. This is mainly due to the mechanical difficulty of main- taining a fixed position and rotation speed of the four rollers, which results in the variation of the stagnation point position up to a typical value of $50 \mu \mathrm{m}$. For the flow in the cross-slot microchannel, variations were limited to $1 \mu \mathrm{m}$.

Successfully carrying out the micro-PIV experiments relied on removing the bias error from misalignment of the camera. Removing this error led to a reduction in the deviation from an ideal straining flow, from $4 \%$ to $2 \%$ for all of the flow rates at the central region of $0.6 w \times 0.6 w$. Therefore, the central region of $0.6 w \times 0.6 w$ was identified as the uniform strain rate region with the variation of $2 \%$.

The strain rate and flow rate were found to be related by a linear relationship. The cross-slot channel flow reached a strain rate of up to $142 \mathrm{~s}^{-1}$ at a high flow rate, whereas $6 \mathrm{~s}^{-1}$ was the highest reported strain rate in four-roll mills.

\section{Acknowledgements}

The authors gratefully acknowledge the Australian Research Council for the financial support of this work. This work was performed in part at the Melbourne Centre for Nanofabrication (MCN) in the Victorian Node of the Australian National Fabrication Facility (ANFF).

\section{Appendix A}

\section{Micro-PIV processing}

To tackle the common issues in micro-PIV recordings, i.e. low density images with strong background noise, the following image processing techniques were applied.

\section{A.1 Removing background noise}

In order to remove the background noise involved, we ensemble-averaged 100 images in the first exposure and 100 images in the second exposure. This was followed by subtracting the corresponding ensemble-averaged images from each image in the first exposure and the second exposure. Figures A.1a and A.1b show an example of an image before 


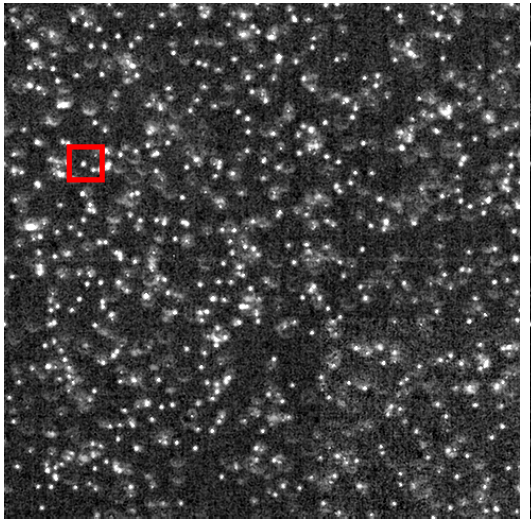

(a) No pre-processing.

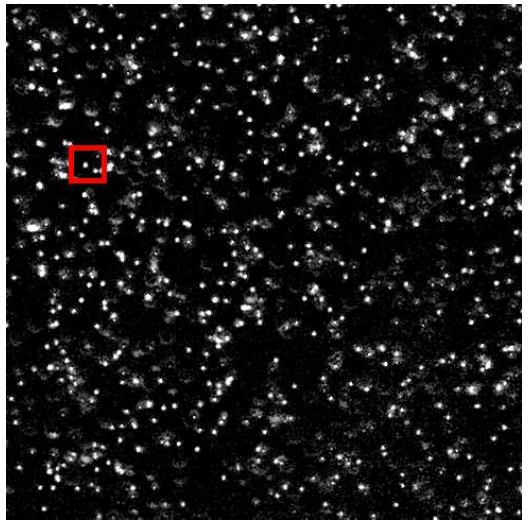

(b) After background noise removal.

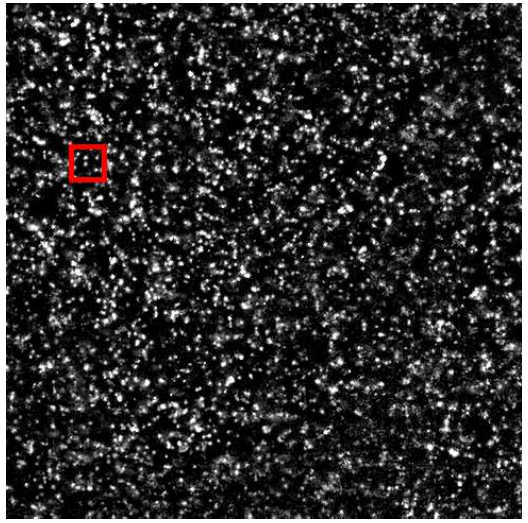

(c) After overlaying six LID-PIV recordings.

Figure A.1: An example of the cropped micro-PIV recordings of the central region of the cross-slot junction in the first laser exposure. The red square represents a $32 \times 32$ pixel interrogation window zoomed in figure A.2.

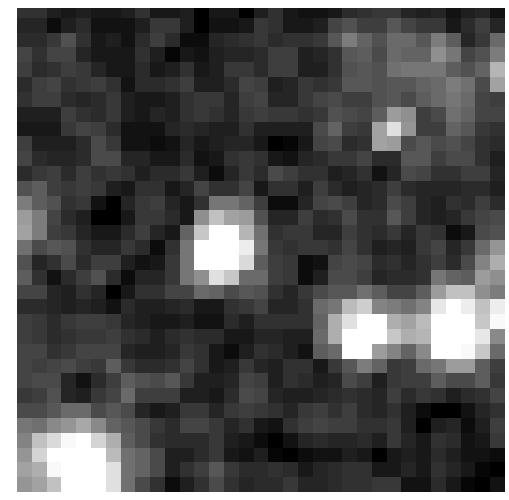

(a) No pre-processing

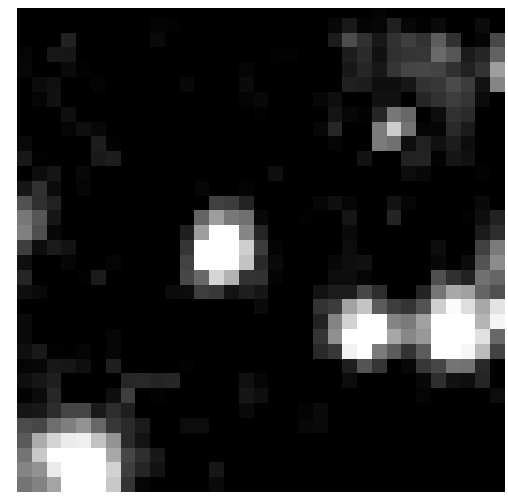

(b) After background noise removal.

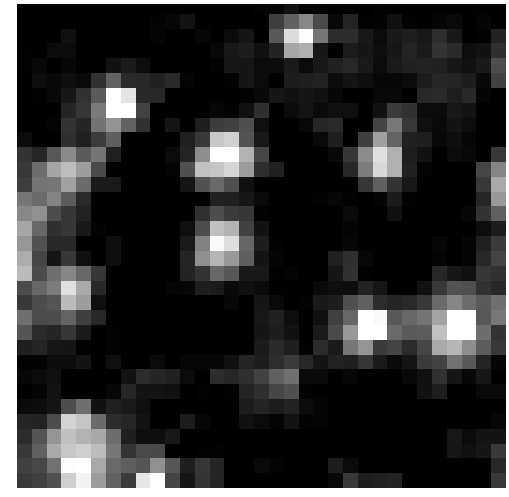

(c) After overlaying six LID-PIV recordings.

Figure A.2: The $32 \times 32$ pixel interrogation window of the figure A.1 that is shown the red square.

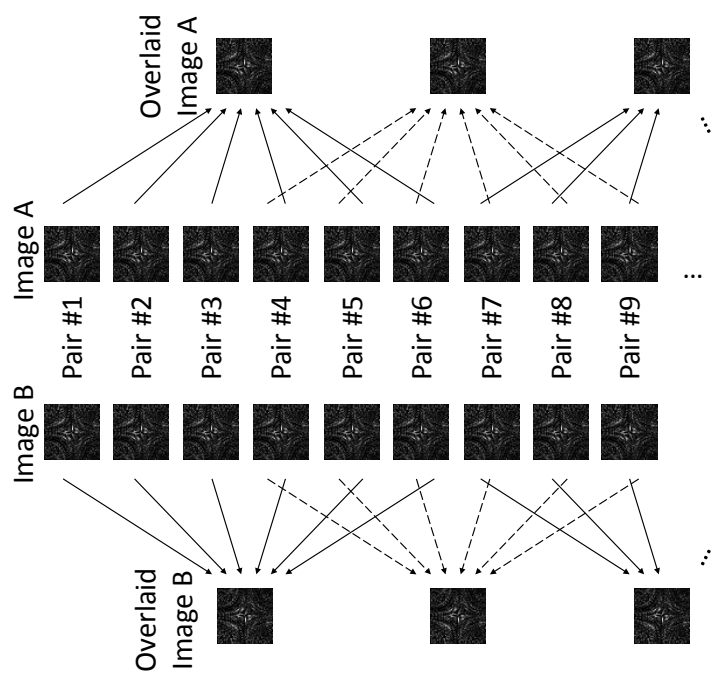

Figure A.3: Overlaying LID-PIV recording algorithm: overlaying six images in the first exposure with $50 \%$ overlap, and the same operation for the images in the second exposure, to obtain a new high density image.

and after background noise removal. An interrogation window of $32 \times 32$ pixels was selected from the same position (red square) in figures A.1a and A.1b and they are shown in figures A.2a and A.2b.

\section{A.2 Averaging method. Step 1 - Av- erage Image Method}

In micro-PIV, seeding density is minimised to reduce the noise produced by the out-of-focus particles. When the seeding density is too low, the standard PIV cross-correlation methods fail to produce sufficient signals to obtain accurate and reliable results. To tackle the low image density (LID) issue, digital image processing techniques are commonly applied to artificially increase the number of particles per image. There are three common techniques to alleviate this problem: "Average Image Method", "Average Velocity Method" and "Average Correlation Method". Using the Average Velocity Method is usually not recommended 


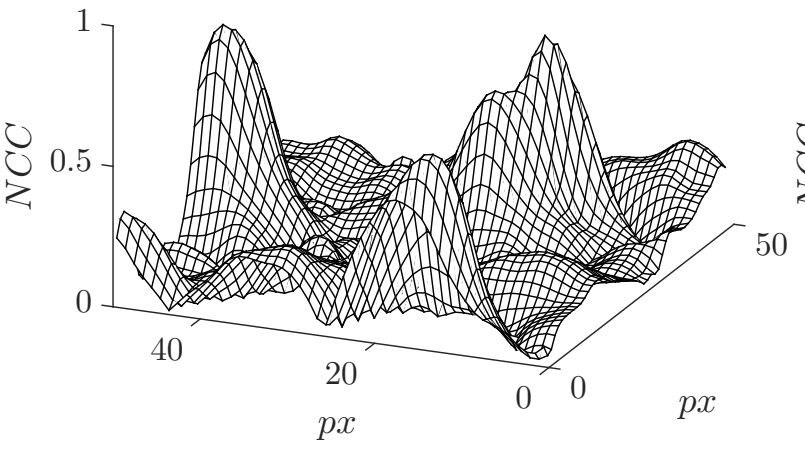

(a) No pre-processing.

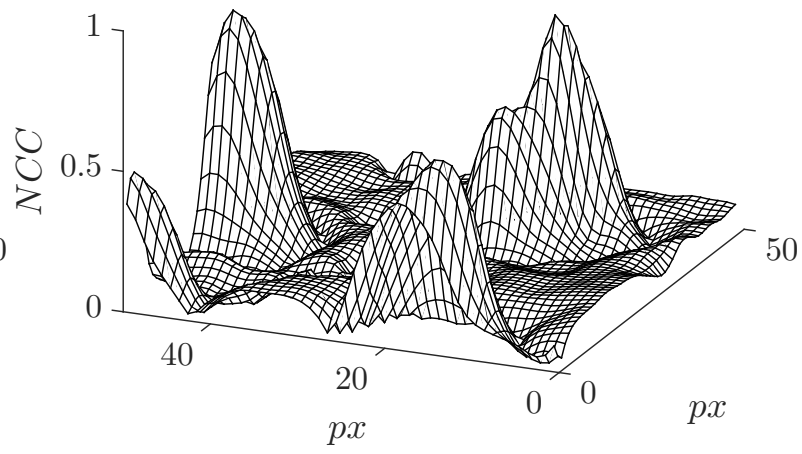

(b) Background noise removal.

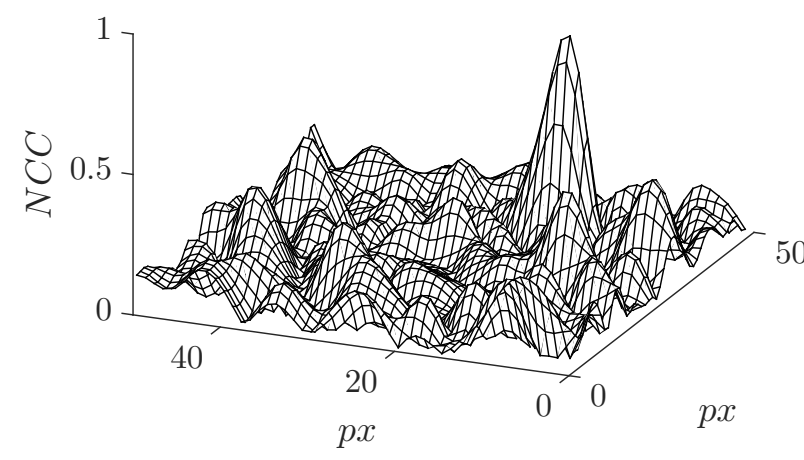

(c) Overlaying of six LID-PIV recordings.

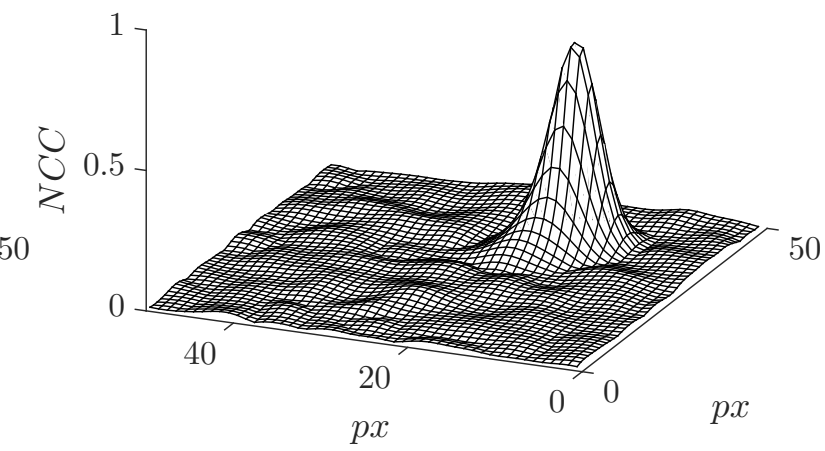

(d) Averaging of correlation functions over $\mathrm{N}>500$ realisations.

Figure A.4: Normalised Cross-Correlation (NCC) coefficient: A qualitative comparison of the signal-to-noise ratio and detectability of a typical selected interrogation window for pre-processing and time-averaging methods.

as all the instantaneous velocity vectors must be valid before averaging, which is often not the case [48]. The Average Image Method is limited in accuracy. This limitation stems from the fact that if too many images are added together, the visibility of the particles is diminished. The Average Correlation Method is known as the superior method compared to the other methods [49]. It is however, not possible to study time-resolved data when this method is employed. In this study a combination of the Average Image Method and the Average Correlation Method was used.

The averaging techniques can legitimately be applied in micro-PIV experiments because the Reynolds numbers in the microchannels are very low, and the flow can be considered laminar and steady or periodic. However, we are also interested in studying the steadiness of the position of the stagnation point. This enables us to investigate the issue of fluctuations in the location of the stagnation point.

Firstly, the Average Image Method was applied, i.e. overlaying of six LID-PIV images with $50 \%$ overlap to satisfy Nyquist criterion (table 3 ). The algorithm is graphically illustrated in figure A.3. This enables us to reach approximately 9.5 particles per interrogation window on average (ideal for PIV measurements), compared to about three particles per interrogation window before the ensemble averaging. Figures A.1 and A.2 show increases in the numbers of particles per image and interrogation window by comparing figure A.1c and A.2c to A.1b and A.2b, respectively.

The above procedure allows for the possibility of studying the time-resolved velocity field. However, instead of each instantaneous velocity field, which was originally spaced at $1 / 15 \mathrm{sec}$, we have timeaveraged fields spaced at $3 / 15$ seconds. 


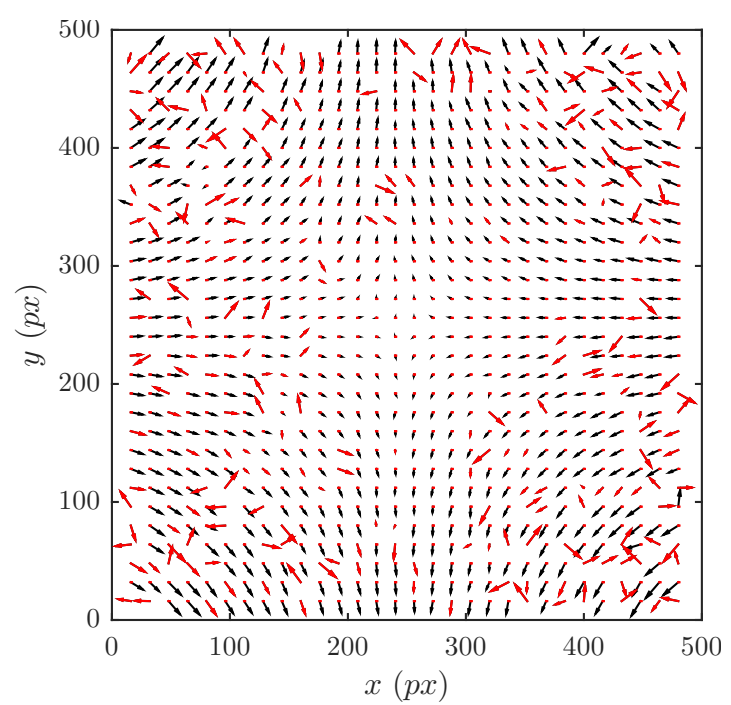

$\longrightarrow$ Diplacement vector $\longrightarrow$ Spurious vectors

(a) No averaging.

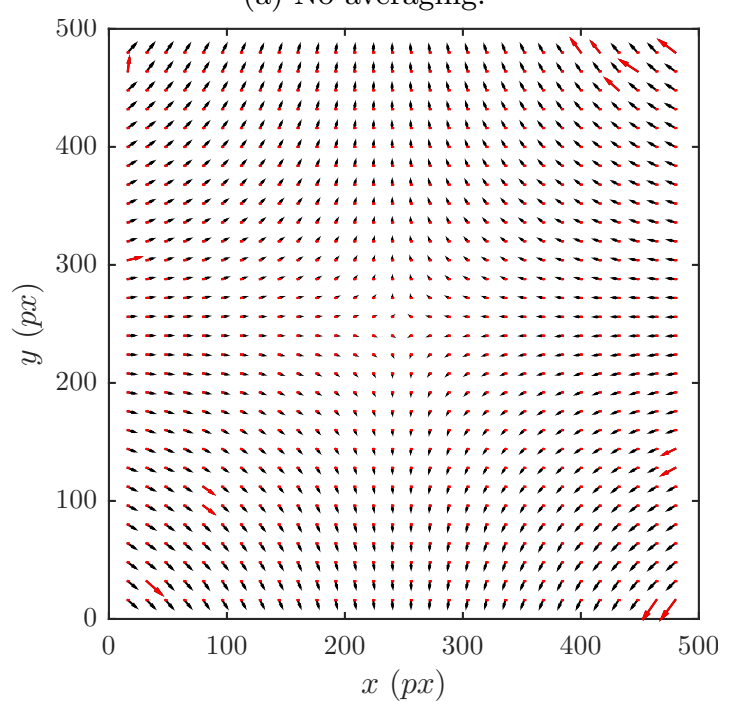

$\longrightarrow$ Diplacement vector $\longrightarrow$ Spurious vectors

(c) Overlaying of six LID-PIV recordings with multipass spurious treatment.
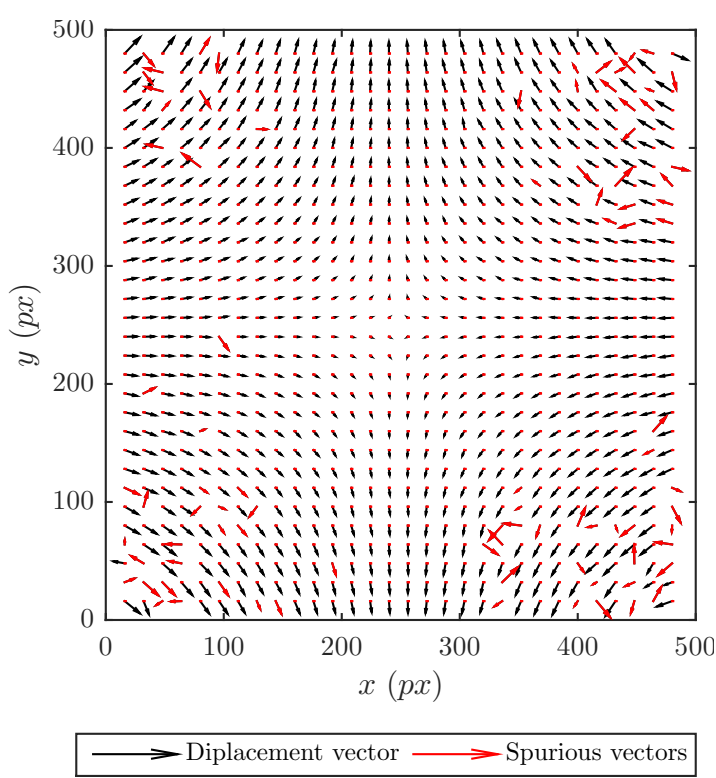

(b) Overlaying of six LID-PIV recordings.

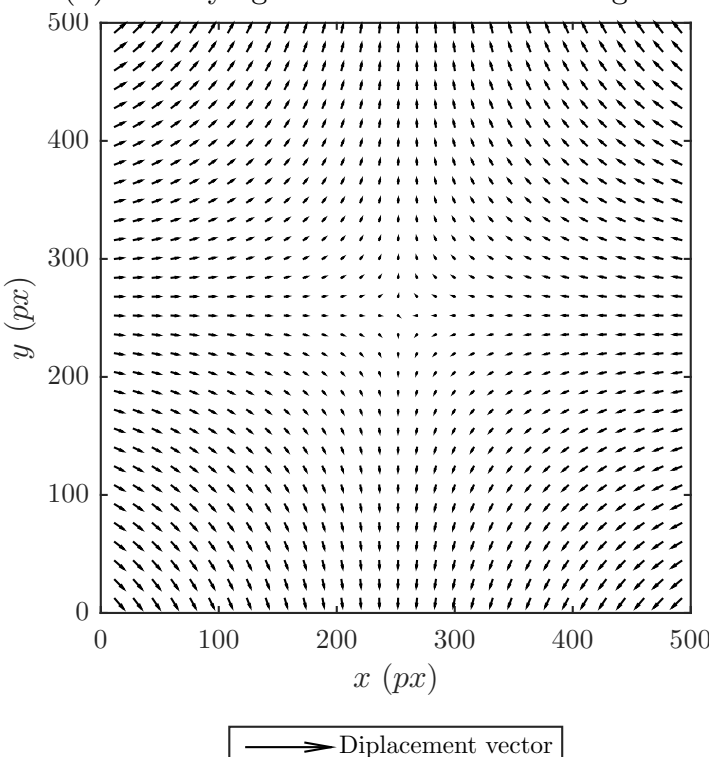

(d) Averaging of correlation map over N >500 realisations.

Figure A.5: Valid (black) and spurious (red) local displacement vectors in pixels over the central region of $500 \times$ 500 pixels.

\section{A.3 Interrogation strategy}

A multigrid algorithm with adaptive central difference interrogation (CDI) offsetting was employed, in which the interrogation window size is successively reduced to the desired resolution (the parameters are listed in table 3 ). The multigrid technique was combined with window deformation algorithm $[40,41]$ to reduce the random error caused by deformation of the measured flow.

\section{A.4 Averaging method. Step 2 - Av- erage Correlation Method}

After applying the first averaging method and correlating the images, the Average Correlation Method was applied to obtain a more accurate velocity field and strain rate. In this technique the correlations were averaged over $\mathrm{N}>500$ realisations. Each measurement was carried out over four minutes and after applying the Average Image Method, $\mathrm{N}=1198$ realisations were achieved. However, averaging correlations over the first 500 reali- 
sations was sufficient for the convergence of the correlation map. To reach sub-pixel accuracy (to avoid pixel locking), a "Gaussian fit" estimator (that yields the best result amongst other three-point estimators, such as "Centroid" and "Parabolic fit") was fitted to the correlation peak [50]. As previously mentioned, the local displacement obtained from each interrogation window is determined by the location of the correlation peak. Therefore, an increase in probability of the correlation peak detection leads to a decrease in the probability of unrealistic/spurious velocity existence. To determine the probability of the detection of the tallest peak, "peak detectability" $\left(D_{0}\right)$ is defined as the ratio of the amplitude of the tallest peak to the second tallest peak in correlation function [51].

To compare the effect of the pre-processing techniques and averaging methods on the detectability and signal-to-noise ratio, the correlation map of a typical interrogation window is plotted in figure A.4. In figure A.4a the detectability $\left(D_{0}\right)$ is about one, which means the probability of detecting the correlation function is close to zero. Upon applying the background noise removal method, the level of noise was decreased, however, still a very low level of signal-to-noise ratio can be seen in figure A.4b. Once the Average Image Method was employed the detectability increased to about three times its initial value (figure A.4c). It should be noted that although this was a typical example of an interrogation window, in some, the detectability was still too low to produce good results. This issue is investigated in section A.5. Finally, by employing the Average Correlation Method over 500 realisations, an ideal correlation map with a distinct peak $\left(D_{0} \approx 50\right)$ was obtained (figure A.4d).

\section{A.5 Spurious vector treatment}

The normalised median test, proposed by Westerweel and Scarano [52], was used to detect outlier data. In this method, velocity vectors are identified as outliers if there is a large deviation with respect to the adjacent data points. Here, the acceptable noise level and the data point neighbourhood radius were set to 0.1 and 1 pixel, respectively. In the case of using the Average Image Method for each semi- instantaneous datum, the normalised median test was employed to detect the spurious vectors, which were corrected using second order correlation.

Figure A.5 shows the effect of the averaging methods on the number of detected spurious vectors. On average, more than $30 \%$ of vectors were labelled as invalid data when no averaging technique was used. Figure A.5a is an example of the displacement vector field for this case. Once the six LID-PIV recordings were overlaid, the number of invalid vectors markedly decreased to less than $8 \%$ on average (figure A.5b - before any spurious correction). By replacing the invalid data (figure A.5c) the number of spurious velocity data points reduced to less than $0.05 \%$ and the displacement vector fields became very smooth, similar to a linear velocity field flow. Finally, after applying the Average Correlation Method no spurious vectors were detected (figure A.5d).

\section{Appendix B}

\section{Potential flow simulation}

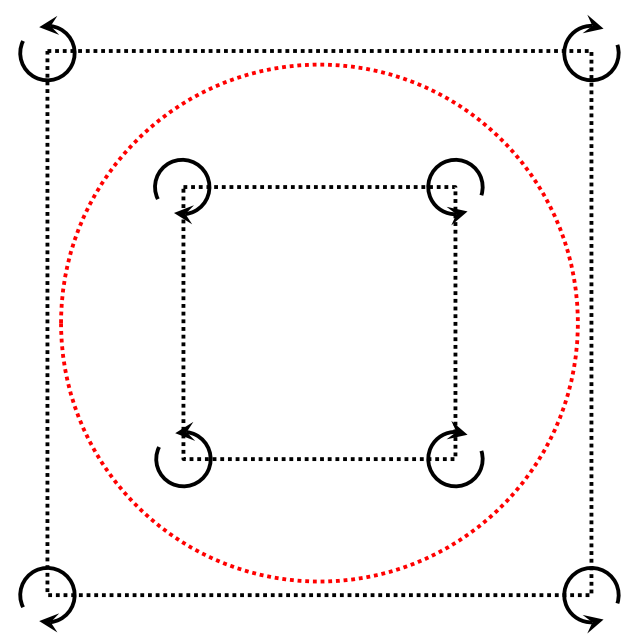

Figure B.1: Arrangement of the eight point vortices in the potential flow to generate the flow in four-roll mill. the dotted red line shows the zero velocity region corresponding to the wall of the container.

To simulate the flow in the whole cell of the fourroll mill by a potential flow, eight point vortices of circulation $\Gamma_{v}$ were super-positioned with the arrangement shown in figure B.1. The inner four vortices are responsible for generating the extensional flow between the rollers, while the others simulate 
the wall of the container (i.e. mirror method in potential flow). The circulation of the point vortices was computed using the angular speed of rollers $\left(r \Omega=\frac{\Gamma_{v}}{2 \pi r}\right)$. Figure B.2 shows the flow streamlines obtained from the potential flow simulation, which is similar to the flow in the whole cell of a four-roll mill.

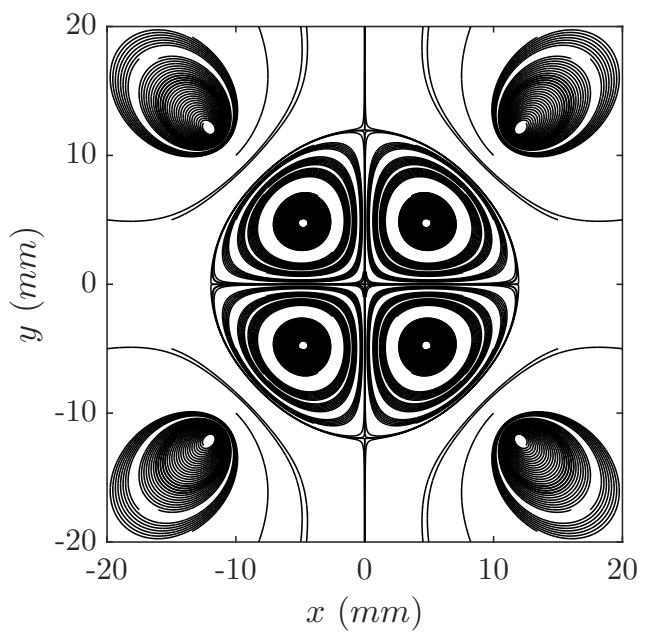

Figure B.2: Flow streamlines in the four-roll mill obtained from the potential flow.

To generate eccentric motion of the rollers, the four inner vortices were moved on a circle around their initial position. Although this type of movement is not exactly the same as the eccentric rotation of the rollers, it can estimate how the stagnation point can be affected when the rollers wobble.

\section{Appendix C}

\section{Removing the bias error}

To provide more information regarding the increase in the deviation from the linear velocity field, variations of $u$ at three different $x=$ constant locations and the variation of $v$ at three different $y=$ constant locations are plotted in figure C.2. To investigate the velocity profile of the various flow rates, nondimensional parameters are defined as:

$$
\begin{gathered}
x^{*}=\frac{x}{w}, \quad y^{*}=\frac{y}{w}, \\
u^{*}=\frac{u}{\gamma w}, \quad v^{*}=\frac{v}{\gamma w} .
\end{gathered}
$$

From the data provided in figure C.2, it can be

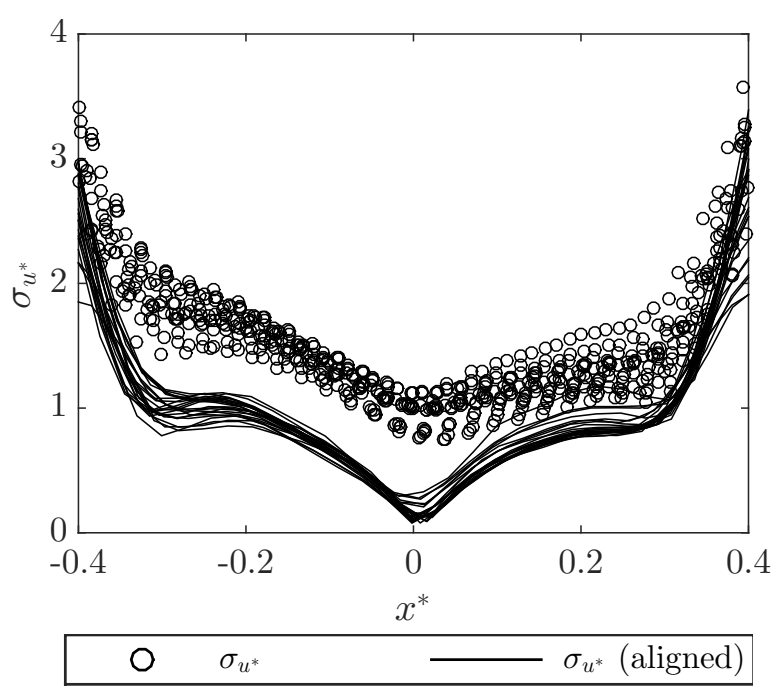

(a) The standard deviations of $u^{*}$

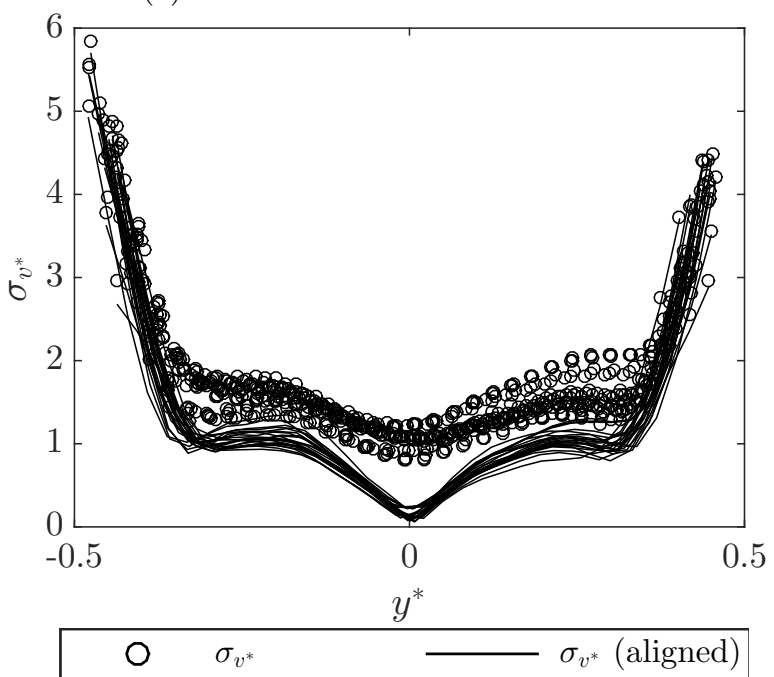

(b) The standard deviations of $v^{*}$.

Figure C.1: The standard deviations of $u^{*}$ and $v^{*}$ were reduced to half of their initial values by tilting the velocity field and removing the bias error due to the camera misalignment.

seen that $u$ and $v$ for all flow rates collapsed at each location, however, the velocity profiles at different locations vary from the linear expected trend. The velocity field at the locations closer to the centrelines of the channels show a more linear behaviour. A closer look at the $x^{*}=0$ or $y^{*}=0$ locations shows a discrepancy between the slope of the measured velocity and the ideal linear velocity. This suggests an angular misalignment of the camera and the microchannel. To address this problem, the angle between $x^{*}=y^{*}$ and $u^{*}\left(x^{*}=0\right)$ was determined for different flow rates and it was found that the maximum misalignment angle is about $1.5^{\circ}$. Based on this misalignment angle, the whole veloc- 


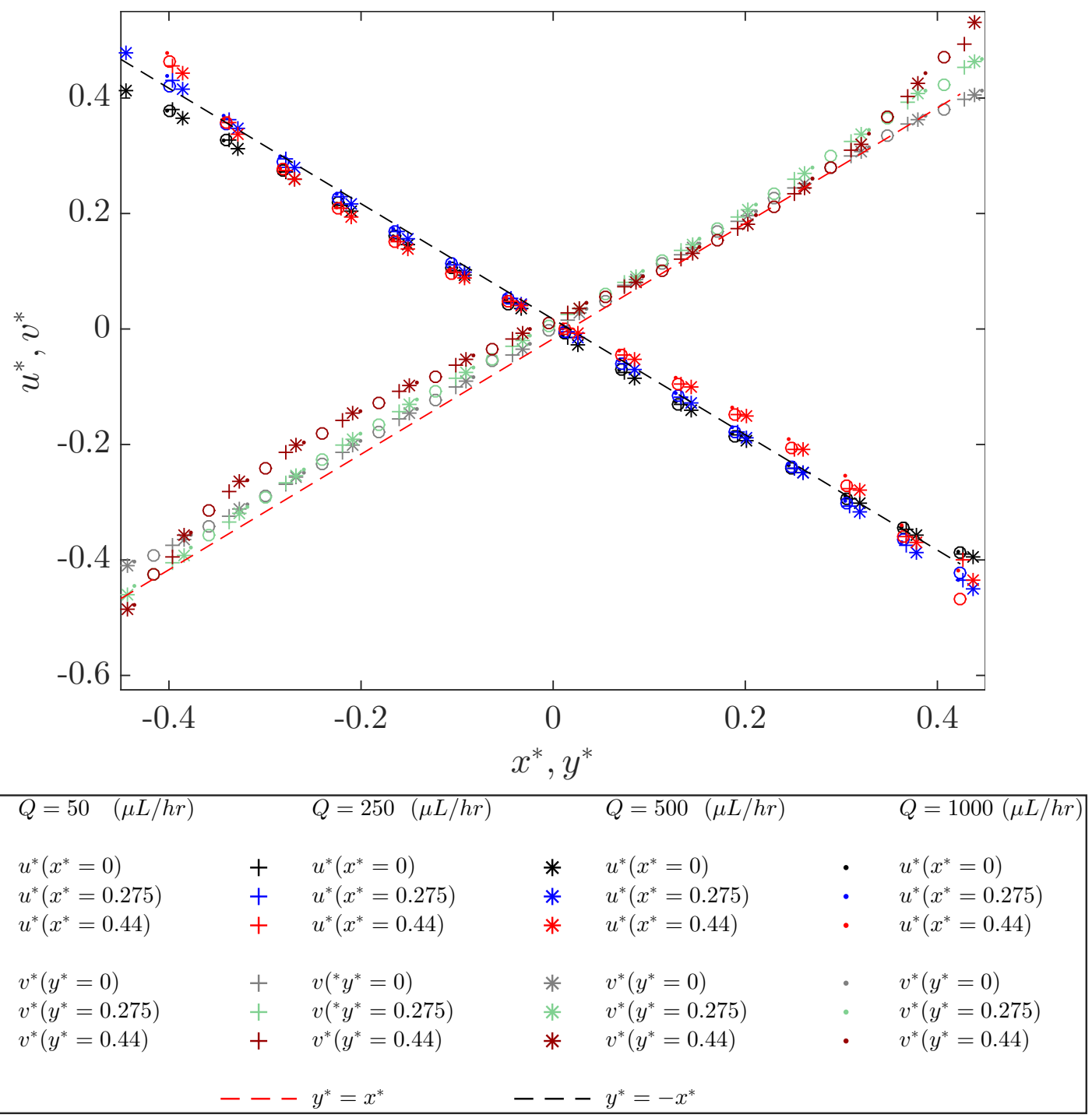

Figure C.2: Comparison of the measured $u$ and $v$ velocity components at different $x^{*}=$ constant and $y^{*}=$ constant locations with ideal linear velocity field, at different flow rates.

ity field was tilted around the intersection of these two lines.

To study the effect of this rotation, the initial values of standard deviations of the $u^{*}$ and $v^{*}$ and their values after rotation are represented in figure C.1. It should be noted that figure C.1 shows the same values as the error bars in figure 15. Therefore, the bias error in the deviation of the measured velocity from the ideal linear flow was removed from the data and the deviations reduced to half of their initial values. It can also be concluded that for all the flow rates, the deviation from a uniform straining flow in the central region $(-0.6 w<x, y<0.6 w)$ was less than $2 \%$. In other words, in this region the relation $\frac{d u}{d x}=\frac{d v}{d y}=\gamma$ is held with the accuracy of $2 \%$. 


\section{References}

[1] Boger D 1987 Annu. Rev. Fluid Mech. 19 157182

[2] Yarin A L 1993 Free liquid jets and films: hydrodynamics and rheology (Longman Publishing Group)

[3] Taylor G I 1934 Proc. R. Soc. Lond. A 146 501-523

[4] Tanyeri M, Johnson-Chavarria E M and Schroeder C M 2010 Appl. Phys. Lett. 96 224101

[5] Lagnado R R, Phan-Thien N and Leal L 1984 Phys. Fluids 27 1094-1101

[6] Lagnado R, Phan-Thien N and Leal L $1985 \mathrm{~J}$. Non-Newton. Fluid Mech. 18 25-59

[7] Lagnado R R and Leal L G 1990 Exp. Fluids 9 25-32

[8] Higdon J 1993 Phys. Fluids 5 274-276

[9] Andreotti B, Douady S and Couder Y $2001 \mathrm{~J}$. Fluid Mech. 444 151-174

[10] Bentley B and Leal L G 1986 J. Fluid Mech. $167241-283$

[11] Milliken W and Leal L 1991 J. Non-Newton. Fluid Mech. 40 355-379

[12] Ramaswamy S and Leal L 1999 J. NonNewton. Fluid Mech. 85 127-163

[13] Innings F, Hamberg L and Trägårdh C 2005 Chem. Eng. Sci. 60 4771-4779

[14] Philip J and Cohen J 2006 J. Fluid Mech. 555 459-473

[15] Dean W 1928 Lond. Edinb. Dubl. Phil. Mag. 5 673-695

[16] Ulloa C, Ahumada A and Cordero M L 2014 Phys. Rev. E 89033004

[17] Tanyeri M, Ranka M, Sittipolkul N and Schroeder C M 2011 Lab Chip 11 1786-1794
[18] Tanyeri M and Schroeder C M 2013 Nano Letters 13 2357-2364

[19] Johnson-Chavarria E M, Tanyeri M and Schroeder C M 2011 J. Vis. Exp. e2517-e2517

[20] Johnson-Chavarria E M, Agrawal U, Tanyeri M, Kuhlman T E and Schroeder C M 2014 Lab Chip 14 2688-2697

[21] Kantsler V and Goldstein R E 2012 Phys. Rev. Lett. 108038103

[22] Xu W and Muller S J 2011 Lab Chip 11 435442

[23] Li Y, Hsiao K W, Brockman C A, Yates D Y, Robertson-Anderson R M, Kornfield J A, San Francisco M J, Schroeder C M and McKenna G B 2015 Macromolecules 48 59976001

[24] Gossett D R, Henry T, Lee S A, Ying Y, Lindgren A G, Yang O O, Rao J, Clark A T and Di Carlo D 2012 PNAS 109 7630-7635

[25] Henry T, Gossett D R, Moon Y S, Masaeli M, Sohsman M, Ying Y, Mislick K, Adams R P, Rao J and Di Carlo D 2013 Sci. Transl. Med. 5 212ra163-212ra163

[26] Perkins T T, Smith D E and Chu S 1997 Science 276 2016-2021

[27] Latinwo F, Hsiao K W and Schroeder C M 2014 J. Chem. Phys. 141174903

[28] Shenoy A, Tanyeri M and Schroeder C M 2015 Microfluid. Nanofluidics 18 1055-1066

[29] Cha S, Shin T, Lee S S, Shim W, Lee G, Lee S J, Kim Y and Kim J M 2012 Anal. Chem. 84 10471-10477

[30] Henon Y, Sheard G J and Fouras A 2014 RSC Advances 4 36079-36088

[31] Pathak J A and Hudson S D 2006 Macromolecules 39 8782-8792

[32] Alicia T G, Yang C, Wang Z and Nguyen N T 2016 Lab Chip 16 368-376 
[33] Dylla-Spears R, Townsend J E, Jen-Jacobson L, Sohn L L and Muller S J 2010 Lab Chip 10 1543-1549

[34] Thomas W H and Gibson C H 1990 J. Appl Phycol. 2 71-77

[35] Paerl H W and Tucker C S 1995 J. World Aquac. Soc. 26 109-131

[36] Moisander P H, Hench J L, Kononen K and Paerl H W 2002 Limnol. Oceanogr. 47 108119

[37] Hondzo M and Wuest A 2008 Environ. Sci. Technol. 43 764-768

[38] Fogg G et al. 1960 Proc. R. Soc. Lond., B, Biol. Sci. 153 111-127

[39] Lee S S, Yim Y, Ahn K H and Lee S J 2009 Biomed. Microdevices 111021

[40] Huang H T, Fiedler H E and Wang J J 1993a Exp. Fluids 15 168-174

[41] Huang H T, Fiedler H E and Wang J J 1993b Exp. Fluids 15 263-273

[42] Akbaridoust F, Philip J and Marusic I 2016 Proc. 20th AFMC. Conf.
[43] Olsen M G and Adrian R J 2000 Exp. Fluids 29 S166-S174

[44] Santiago J G, Wereley S T, Meinhart C D, Beebe D J and Adrian R J 1998 Exp. Fluids 25 316-319

[45] Meinhart C D, Wereley S T and Santiago J G 1999 Exp. Fluids 27 414-419

[46] Meinhart C D, Wereley S T and Gray M H B 2000 Meas. Sci. Technol. 11809

[47] Prasad A K, Adrian R J, Landreth C C and Offutt P W 1992 Exp. Fluids 13 105-116

[48] Meinhart C D, Wereley S T and Santiago J G 2000 J. Fluids Eng. 122 285-289

[49] Wereley S T, Gui L and Meinhart C D 2002 AIAA J. 40 1047-1055

[50] Adrian R J and Westerweel J 2011 Particle image velocimetry (Cambridge University Press)

[51] Coupland J M and Halliwell N A 1988 Appl. Opt. 27 1919-1921

[52] Westerweel J and Scarano F 2005 Exp. Fluids 39 1096-1100 